\title{
Highly Accurate, Reliable and Non-Contaminating Two-Dimensional Material Transfer System
}

\author{
Chandraman Patil(1), Hamed Dalir(1) (2), Jin Ho Kang ${ }^{(3)}$, Albert Davydov(4), Chee Wei Wong( ${ }^{(3)}$, Volker J. \\ Sorger ${ }^{(1)(2)^{*}}$ \\ (1)Department of Electrical and Computer Engineering, George Washington University, Washington, DC \\ 20052, USA \\ (2)Optelligence LLC, 300 Delaware Ave Ste 210-A, Wilmington, DE 19801, USA \\ (3)Fang Lu Mesoscopic Optics and Quantum Electronics Laboratory, University of California, Los Angeles, \\ CA 90095, USA \\ ${ }^{(4)}$ Materials Science and Engineering Division National Institute of Standards and Technology Gaithersburg, \\ MD, USA \\ *Corresponding Author: sorger@gwu.edu
}

\begin{abstract}
The exotic properties of two-dimensional (2D) materials and 2D heterostructures, built by forming heterogeneous multi-layered stacks, have been widely explored across several subject matters following the goal to invent, design, and improve applications enabled by 2D materials. To successfully harvest these unique properties effectively and increase the yield of manufacturing $2 \mathrm{D}$ materialbased devices for achieving reliable and repeatable results is the current challenge. The scientific community has introduced various experimental transfer systems explained in detail for exfoliated 2D materials, however, the field lacks statistical analysis and the capability of producing a transfer technique enabling; i) high transfer precision and yield, ii) cross-contamination free transfer, iii) multi-substrate transfer, and iv) rapid prototyping without wet chemistry. Here we introduce a novel 2D material deterministic transfer system and experimentally show its high accuracy, reliability, repeatability, and non-contaminating transfer features by demonstrating fabrication of 2D material-based optoelectronic devices featuring novel device physics and unique functionality. The system paves the way towards accelerated $2 \mathrm{D}$ material-based device manufacturing and characterization. Such rapid and material-near prototyping capability can accelerate not only layered material science in discovery but also engineering innovations.
\end{abstract}

\section{Keywords}

Two-dimensional materials, transition metal dichalcogenide, light-matter interaction, viscoelastic microstamping, deterministic transfer, reliable and robust system, graphene, hexagonal boron nitride, molybdenum disulfide, molybdenum ditelluride, tungsten selenide, indium selenide, photodetector, cavity enhancement, pn junction. 


\section{Introduction}

Designing novel materials to improve the quality of life is appealing, but it is also challenging in practice. A generic solution for combining the best of different elements in one ultimate substance is still intricate and unsolved. Composite materials and III-V heterostructures have revolutionized many aspects of modern technologies laying the initial milestone in the process of engineering the material properties. However, it is still challenging and complex to mix and match crystalline materials with their unique properties of forming heterostructures with controlled tunning of attributes, functionalities, and properties. Developing novel techniques for using such materials like two (2D) dimensional materials is crucial for further material characterization enabling novel technological advancements.

The mechanical exfoliation of atomically thin 2D graphene layers from its bulk crystals in 2004 [1] introduced a unique research study of two-dimensional materials. This influenced the scientific community to investigate the fundamental graphene properties and applications in various domains [2], [3]. The invention of the micromechanical exfoliation method was the key to the success of graphene science, providing extremely high-quality samples and arousing curiosity for studying other 2D materials like transition metal dichalcogenides (TMDCs), MXenes, and 2D layered material-based insulators [4]-[11]. The exfoliation process produces crystal flakes of various sizes and thicknesses that are non-uniformly (i.e., spatially and material quality) and densely distributed over the target substrate leading to a low yield of useful atomically thin flakes. The hence required implementation of the optical identification method facilitates identifying useful atomically thin flakes on the substrate based on contrast difference in the image for different thicknesses allowing accurate and non-contact techniques of locating the flakes [12]. This method, however, is not suitable for fabricating complex systems such as heterogeneous integration of 2D materials on other pre-fabricated devices and structures [8] or building van der Waals (vdW) heterostructures by artificially stacking different 2D material flakes [13]-[18]. Therefore, it is important to develop new experimental setups and techniques for using 2D materials to fabricate more complex systems with enhanced reliability and functionality. Recently unique and modified techniques are developed such as the pick-and-place approach [19], the wedging method [20], the polyvinyl alcohol (PVA) method [21], patterned stamping method [22], viscoelastic stamping [23], and Evalcite method [24] for deterministic transfer of 2D materials. Each of these helps transfer 2D materials efficiently but is limited by their shortcomings and scope of implementation, such as obtaining a high yield of quality flakes, avoiding contamination of entire substrate from undesired flake transfer, demonstrating repeatability in device performance, and avoiding undesired residues on flakes [13].

The intrinsic properties of $2 \mathrm{D}$ materials are extremely sensitive to the chosen transfer and/or handling method deployed, which can involve temperature-assisted transfer, sacrificial polymer layers, litho-assisted patterned stamps, and/or wet chemical processes for substrate etching in the transfer process. The target substrate surface structures, for example, can be reactive to the chemicals used, the capillary forces involved in the process may crack very thin layers or produce capillary actionassisted undesired strain due to bubbles or wrinkling formed under surface in 
suspended material. 2D materials are also sensitive to temperature changes leading to surface oxidation of materials in few cases. Thus, the dry transfer methods, such as discussed in [22], [23], [25], help in overcoming wet-chemistry related challenges. Despite these modifications in improving the transfer method and system, the critical issue of spatial cross-contamination of the unwanted 2D material crystal flakes on the transfer substrate and yield of good quality flakes are yet to be addressed. An attempt of solving these issues was discussed in [13] focusing on overcoming the crosscontamination challenge in a 2D material transfer system by a comparison-based study. However, with increased research in the field of 2D materials, a reliable, robust, and highly repeatable method and transfer system is important for accelerating the 2D manufacturing process at free space, integrated electronic [26], [27], and/or photonic chip-level, straintronics [28] and large area optics applications.

Here we introduce a 2D material transfer system (herein termed 2DMTS) capable of; i) eliminating contamination on 2D materials-based flakes, devices, and target substrate, ii) increasing the yield and quality of transferred material flakes, iii) enabling rapid and accurate transfer, and iv) achieving without involving wet chemical etching or thermal assisted process step for transfers. 2DMTS is a one-of-a-kind transfer method [13] using a viscoelastic polymer and metal micro-transfer stamper enabling selectivity during transfer. We demonstrate a reliable, robust, and highly accurate method to transfer 2D material onto arbitrary substrates with virtually no crosscontamination showing a high spatial accuracy of $0.5 \pm 0.2 \mu \mathrm{m}$ which is the highest reported to date without cross-contamination. After introducing 2DMTS and experimentally validating its capability and performance limitations, we put its rapid prototyping capability to use demonstrating novel device and fabrication capacities to include building and demonstrating optoelectronic devices featuring device novelty covering the fields of photonics, arrayed structural deposition of materials, heterostructure lattices, and electronic devices, including strainoptronics-based photodetector devices.

\section{Results and Discussion}

The 2D transfer system platform (2DMTS) introduced and discussed here includes a metallurgical microscope, three linear axis stages for movement of Polydimethylsiloxane (PDMS) holder, a micro-transfer-stamper, and a target chip for precise alignment (Fig. 1a). A small screw lock-based clamp needle holder is mounted on an angled arm XYZ axis stage to avoid blocking the field of view of a long working distance microscope mounted on top as seen in Fig. 1a. The micro-stamper can be lowered along Z-axis carefully to touch the substrate and transfer the selected flake after alignment as seen in Fig. 1b. The microscope is mounted with a standard 8-bit CMOS camera. It is used for the alignment of the system and measures the size and area of the flake before the transfer, calibrated for the specific objective lens. This allows for higher 2D material flake selectivity in terms of size and shape as per the requirement of the application. A camera software crosshair is used to align the position of flake, micro-stamper, and target using the respective stages. The soft PDMS helps in transferring material over different structures as it wraps around the profile providing better contact of material with the target without damaging it. The thin (17mil) PDMS sheets (Gel Pak) are cut down to small strips $(1.5 \mathrm{~cm} \times 3 \mathrm{~cm})$ and are mounted on a microscope glass slide based PDMS holder (herein termed as PDMS cartridge). This cartridge is held by clamp mount metal clips keeping the PDMS suspended in the air, mounted on an XYZ axis stage as seen in schematic Fig. 1c. The clamp mount clips help to load and unload the 
PDMS cartridge with ease (quick release) and prevent disturbing the alignment of other parts when changing the 2D material source banks. This allows multiple PDMS strips with different 2D material source banks to be realized for building heterostructures without the need of loading and unloading new PDMS films with different materials. The density of the flakes on Nitto tape is higher after exfoliation from a piece of crystal, forming clusters of un-isolated flakes. While transferring these flakes on a PDMS film, contacting the entire area of PDMS to the tape transfers clusters of 2D material flakes on the PDMS. The lower number of isolated flakes on PDMS leads to lower individual flake transfers from the 2D source bank cartridge. Therefore, it is important to transfer more isolated flakes on PDMS which are loosely adhered to the tape for ease of transfer and increasing the yield. A detailed description of the modified exfoliation of 2D material on PDMS film strips is discussed in the method below. The 2DMTS exfoliation graphical flowchart can be seen in Fig. 1d which shows that the high density of flakes and overlapping clusters formed on the exfoliation tape can be avoided or reduced significantly by using this modified method. The chip or target substrate is cleaned by rinsing with acetone and isopropyl alcohol (IPA) followed by nitrogen drying and is placed on an $X Y-\theta$ stage where the rotation helps to load and unload the substrate for transfer of 2D material.
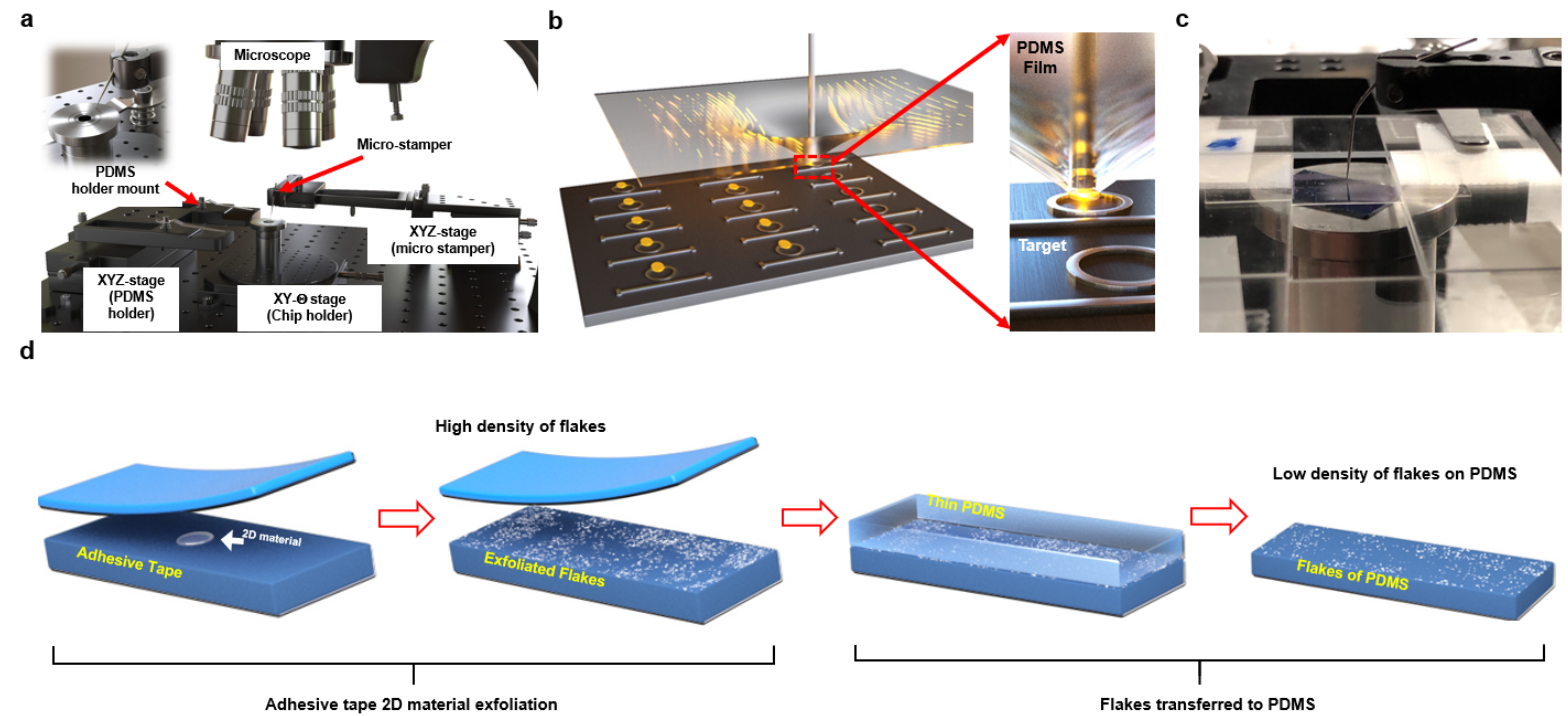

Figure 1. Robust, precise, and reliable 2DMTS. (a) The simplified 2D transfer system platform schematic includes the microscope, linear stages for alignment movement of PDMS holder, micro-stamper, and target substrate, PDMS holder mount clips and, a close view of the micro-stamper mounted on the setup. (b) Schematic of the PDMS, micro-stamper, and target chip position under alignment highlighting the close view of stretched PDMS when micro-stamper is approaching the targeted structure on the chip. Inset: showing the 2D material flake precisely positioned under the tip while the other flakes on the PDMS remain on the noncontact regions of the PDMS on the holder. The magnified view shows the small area of PDMS (largest reported here, $\sim 400 \mu \mathrm{m} \times 200 \mu \mathrm{m}$ ) under the stamper with the flake underneath touching the target. (c) Side angled image of the PDMS holder mounted on the stage with a PDMS strip mounted on it. The PDMS film is positioned over the target substrate and the micro-stamper is then adjusted on top of the selected flake as seen in the image. (d) The modified transfer process of 2D materials on PDMS using the adhesive tape exfoliation method helps to reduce the density of overlapping flakes and provides isolated flakes on PDMS for precisely locating flakes under the microscope. This allows selective transfer of the desired flake without crosscontamination on the chip.

The flake transfer yield is $>(95.0 \pm 0.1) \%$ (Fig. 2), higher than any other state-ofthe-art transfer system so far reported, as the entire surface area of the flake contacts with 
the target forming better adhesion to the target area. The failure in transferring the flake can be caused mainly because of the rough surface of the flake due to improper exfoliation, thus reducing the vdW adhesion strength with the target. A flake larger than the micro-stamper tip size reduces the transfer probability and is prone to break the flakes causing defects. But our different size micro-stamper range ensures flake-stamper size match. The contact area also defines the area of isolation (details in Fig. 3 below) required for each flake on PDMS to avoid multiple flakes being transferred, here solved by using a modified exfoliation process as seen in Fig. 1d to reduce the density of flakes on the PDMS film and discussed further.

The 2DMTS setup has a transfer accuracy of about $0.7 \mu \mathrm{m}$ and a precision of $<100 \mathrm{~nm}$ determined by experimental statistical analysis of the system. This analysis is based on independent experimental transfers of 80 flakes from different 2D materials (graphene, $\mathrm{MoS}_{2}, \mathrm{hBN}$, and $\mathrm{WSe}_{2}$ ) for each stamper size by two different users. Thus, the dependency of a user experience, as observed in other transfer methods, is reduced by analyzing data generated by these two users who helped in the independent transfer of materials for producing data. The flake position distribution after transfer from the crosshair for the three stamper sizes can be seen in Fig. 2a-c where the inset shows the respective micro-stamper side view schematic. The distances of flakes transferred from the target point were measured using the calibrated camera software. The long working distance objective lenses can be switched to different magnifications for; 10x coarse (target search), 20x fine (flake search), and 40x finest (flake transfer) alignment based on the target size. Also, it is necessary to consider the flake size being transferred, therefore, for a wide range (1 um $\sim 150 \mathrm{um}$ ) of flake sizes, largest limited by the exfoliation method, we have built different elliptical tip-sized micro-stampers herein termed as stamper\#1 (425 $\mu \mathrm{mx} 200 \mu \mathrm{m})$, stamper\#2 $(300 \mu \mathrm{m} \times 145 \mu \mathrm{m})$, and stamper\#3 $(185 \mu \mathrm{m} \times 130 \mu \mathrm{m})$ built as discussing further. Details about the micro stamper are shared below. The histogram for the independent transfer attempts for each micro-stamper size shows the small offset, indicated by the peak position involved in the transfer location for varying micro-stamper sizes (Fig. 2d). The associated standard deviation distribution shows the dispersion of the transfer locations for each stamper normalized to the offset (Fig. 2e). Fig. 2f shows the accuracy and precision of each microstamper demonstrating the highest accuracy of $0.7 \mu \mathrm{m}$ and precision of $<100 \mathrm{~nm}$. Such accuracy and precision are vital in integrating the 2D material with highly dense photonic or electronic devices on-chip and nano-sensing applications. The smaller micro-stamper sizes provide higher accuracy and precision due to the small area of contact and lower offset deviation from the target. The maximum size of the flake that can be transferred and the effective area of contact of the PDMS with the three micro-stamper sizes considered in this article can be seen in Fig. $\mathbf{2 g}$. This helps the user to choose the appropriate stamper size for achieving optimized transfer accuracy for desired flake size and target. The inset images represent the effective area of contact of PDMS to the target substrate when each flake is transferred and defines the area of isolation of flakes as well. The image contrast of the PDMS stamping area under the microscope is enhanced by the false color (purple) area for better representation.

For determining the yield of the transfer process produced by this system we transfer flakes of different sizes at different random locations on a $\mathrm{SiO}_{2}$ substrate (Fig.2h). This experimentation is designed to test the reliability and repeatability of the system for multiple single flake transfers that can be achieved in a single attempt. The transfer of the $2 \mathrm{D}$ material 
flake to the substrate without cross-contamination and 2D material deformation cracking of the flake into pieces by any means was termed as a success. The probability distribution of the success and failure rate of the transfer system was calculated using this data set as seen in Fig. $\mathbf{2 h}$. All the 40 independent transfers were made in sequence without making any changes to the PDMS film or setup alignment apart from searching the different flakes on PDMS and changing the target location. Therefore, the setup is proved to be highly repeatable, reliable, precise, and accurate for a wide range of applications that can be developed for science and technological advancements. Such a plethora of system properties has not been achieved under any other 2DMTS as discussed in [13], [19]-[23], [29].

a

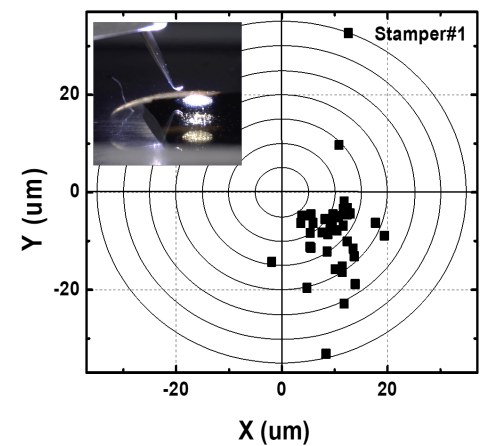

d

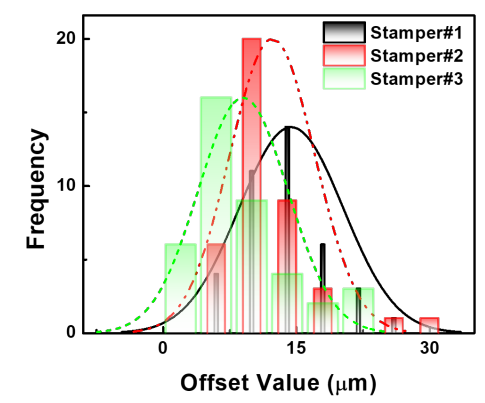

g

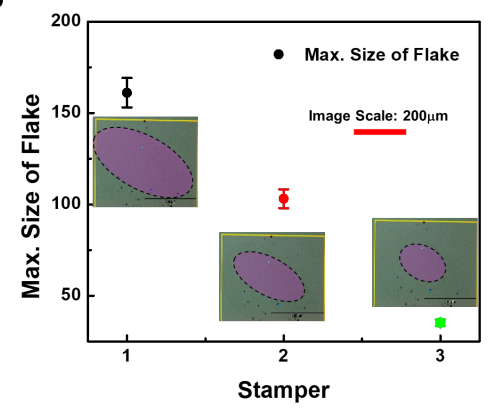

b

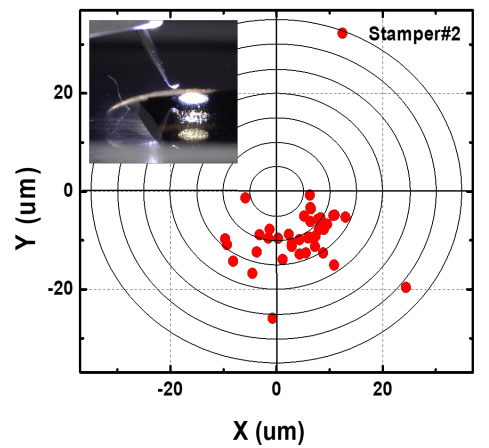

e

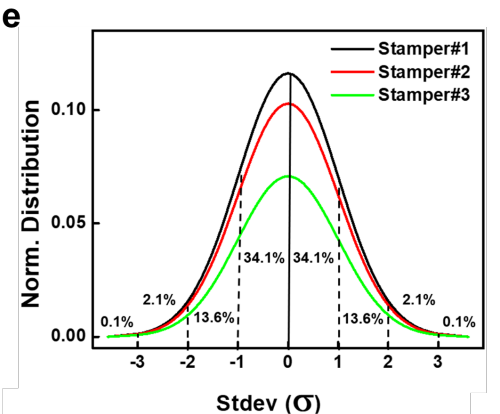

h

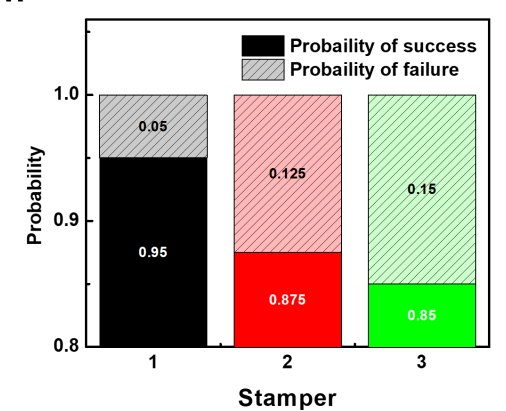

C

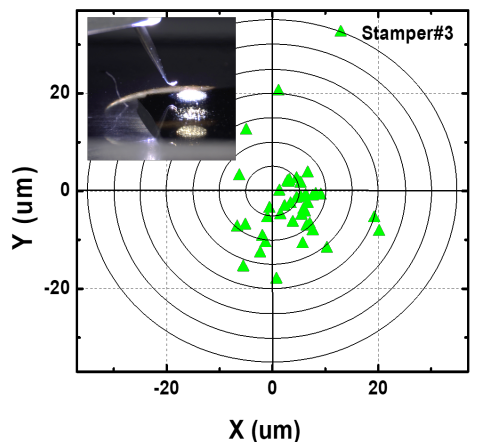

f

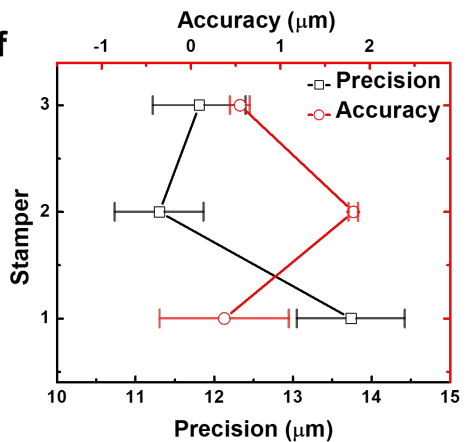

i

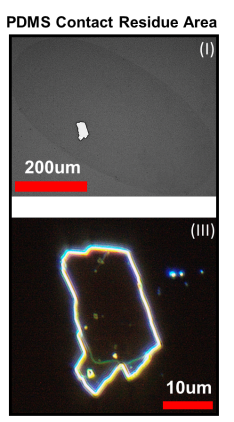

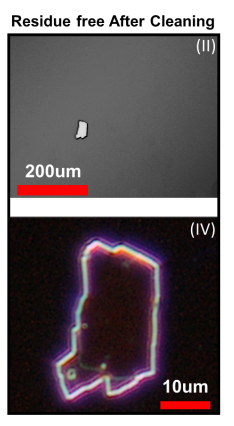

Figure 2. 2D printer setup performance analysis for accuracy, precision, and reliability test. (a)-(c) Distribution of position of flakes transferred on $\mathrm{SiO}_{2}$ substrate measured from the center of the target. The offset produced due to optics and the size of the micro stamper can be observed in the graph. The inset shows the side view image representation of the micro stamper size and shapes positioned on top of the PDMS. (d) Histogram fitted with a gaussian curve that illustrates standard deviation of transferring process for each microstamper size. The graph also represents the offset in target location due to optics and stamper size (e) Standard deviation distribution of the flake transfer position on target measured considering the center of the flake to the software crosshair. Each band has 1 standard deviation, and the labels indicate the approximate proportion of area (note: these add up to $99.8 \%$ of data, and not 100 percent of data because of rounding for 
presentation). (f) The accuracy and precision values with error bars are computed for each stamper showing high accuracy for small stamper size and a reduced accuracy and precision for large stamper size. $(\mathrm{g})$ Maximum size of flake that can be transferred with the three sizes of stamper. The inset microscope image represents the effective area of stamping for each stamper. The false-colored elliptical part highlights the stamping area where the scale bar is $200 \mu \mathrm{m}$ as the stamper shape is elongated in one direction as seen in Fig. 2 (a-c) inset (h) The probability distribution of transfer success and failure rate of the transfer system was calculated by gathering 40 data sets by transferring different flakes in a different location (i) Microscope image of transferred $\mathrm{MoS}_{2}$ flake on oxide substrate with PDMS residue (I) and after cleaning process showing the residue-free area (II). The flake area after the transfer (III) and residue-free area after the cleaning process (IV) is confirmed by darkfield microscopy.

The flake after the transfer is cleared of PDMS residues on top of the flakes after the transfers have been completed. It is useful to consider samples where the material is transferred on a structured profile while rinsing and drying with nitrogen gas with as few vibrations as feasible. The vdW forces are prone to be not strong on the sides of the structures as the flakes may not conformally cover the structures owing to their thickness.

(a)
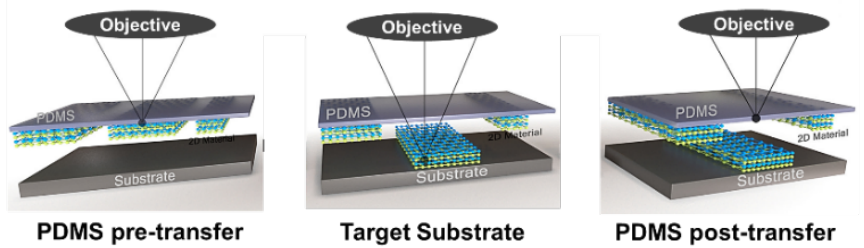

PDMS pre-transfer

Target Substrate

PDMS post-transfer

(b)
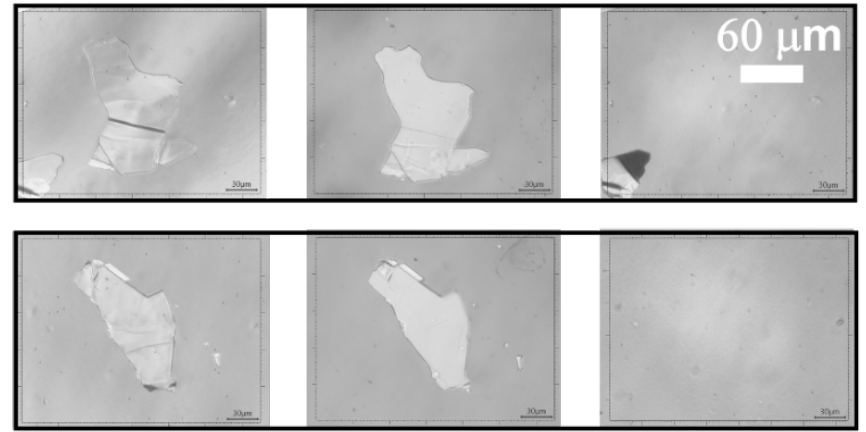

(c)

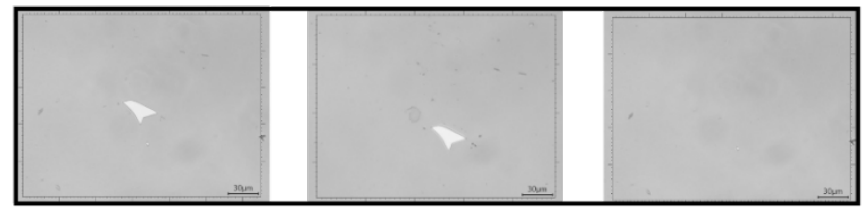

(d)

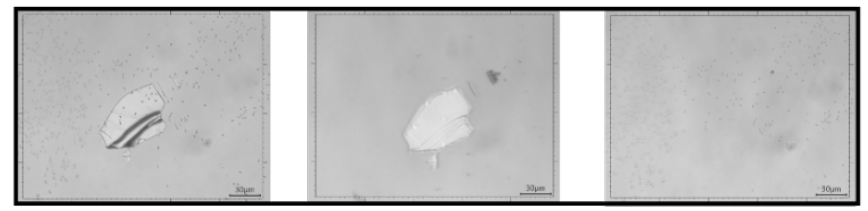

(e)

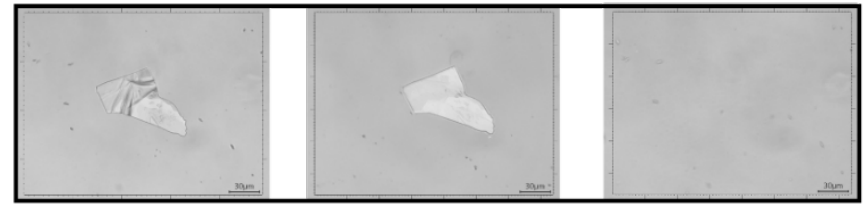

(f)

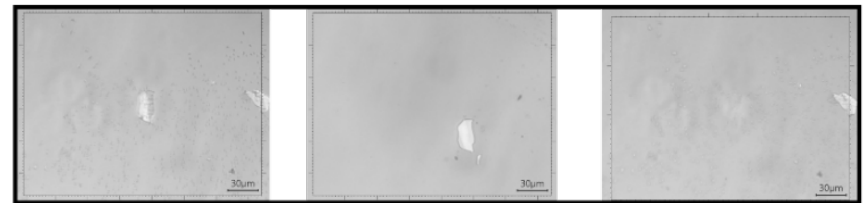


Figure 3. Transfer results from the 2D material transfer system. (a)-(f) Optical microscope images for 2D graphene flake on PDMS (before transfer), a substrate with flake after the transfer, and of PDMS (after transfer) captured while using the 2D printer setup. The images verify no cracking, wrinkles, or folding of the flake after transfer on the substrate.

This prevents the transferred flakes from moving off the surface due to mechanical vibrations. The issue of weak conformality of the flake coverage can be solved by keeping the sample in a vacuum chamber for 20-30 minutes. This strengthens the adhesion of the flake with the structure profile. On the sample, darkfield microscopy, optical filter-based microscopy, or secondary electron microscopy (SEM) can be used to check the results of the cleaning process depending on the resolution of inspection. As seen in Fig. 2i, a molybdenum disulfide $\left(\mathrm{MoS}_{2}\right)$ flake was transferred on $\mathrm{SiO}_{2}$ substrate, and the contact area can be observed using a microscope, but the contrast is very poor for such small particles. Therefore, the area is enhanced by contrast correction which represents the area of contact in Fig. 2i(I). The difference, in contrast, represents the contacted area of PDMS on the substrate and top of the flake. The residues are completely removed after the post-transfer simple cleaning process as seen in Fig. 2i(II). The clean surface of the flake is verified using darkfield microscope images before Fig. 2i(III) and after the cleaning process Fig. 2i(IV). This shows that the quality of the transferred flake is highly preserved along with no crosscontamination of flakes on the entire substrate.

Further, understanding the non-cross contamination process while transferring multiple flakes in different locations on the substrate is necessary for repeatability and reliability demonstration. Also, there are possibilities of producing wrinkles or bubbles under the flake, cracking of large-sized flakes, and accidental transfer of a nearby flake after the transfer process. In Fig.3a-f, different sizes, and shapes of graphene material flakes are transferred on a $\mathrm{SiO}_{2}$ substrate showing the optical images of flake on PDMS, flake transferred on the substrate, and of PDMS at the same location after transfer captured while using the $2 \mathrm{D}$ printer. The isolation of a nearby flake from the target flake while transfer can be observed in Fig. 3a, $\mathbf{f}$ where the flake on the side does not touch the substrate during the transfer process and can be seen left on the PDMS after transfer of the target flake. However, small flakes within the range of few microns cannot be avoided from getting transferred as it is difficult to isolate such small distances from cross-contamination as verified statistically in Fig. $\mathbf{2 g}$ to the stamper size. Further, these images show that the wrinkling or folding of material caused due to the viscoelastic nature of PDMS does not reflect after transfer on the substrate due to slight stretching on PDMS while stamping. This also indicates that the 2D material flakes are not strongly adhered to the PDMS due to the modified flake transfer technique discussed in Fig. 1d making it easy to transfer it on structures as well. Lastly, if small wrinkles are produced after the transfer, the sample can be kept in the high vacuum chamber to remove it.

\section{DMTS Preparation and Device Fabrication Process}

\section{a) Micro-Stamper}

The micro-stamper is fabricated by modifying tungsten electrical probe needles. The needle is placed on the holder at 45 degrees, then lowered perpendicularly, and forced on the metal plate to bend the needle's tip. The amount of force given to the needle to bend the tip determines the size of the tip bent. The curved tip also provides a smooth tip characteristic to the micro stamper, preventing piercing in the PDMS sheet. The size of the stamping area 
is calculated by measuring the PDMS contact area after transferring.

\section{b) Reusable PDMS Holder Preparation}

The PDMS holder serves the purpose of holding multiple PDMS strips (here three) with a choice of having different/same material on each of the PDMS strips. The PDMS holder requires six thin glass slides $(\sim 1.2 \mathrm{~mm})$, Gel-Pak thin PDMS film (17 mils) strip/s, double-sided and single-sided adhesive tapes. The pictorial representation of preparing the PDMS holder is shown in Fig 4. (1-5).

\section{c) Exfoliation Process}

The exfoliation of 2D materials is a widely accepted technique using a weak adhesive Nitto tape discussed in [1]. To transfer the material onto the PDMS film, a pair of tweezers, weak adhesion Nitto exfoliation tape, a ruler, PDMS strips, and the desired 2D material crystals are needed. A small piece of crystal from bulk 2D material is placed on one end of a Nitto tape strip with a width equivalent to that of a PDMS strip. The other end is closed on the crystal and peeled back and forth until a lot of flakes spread on the tape.

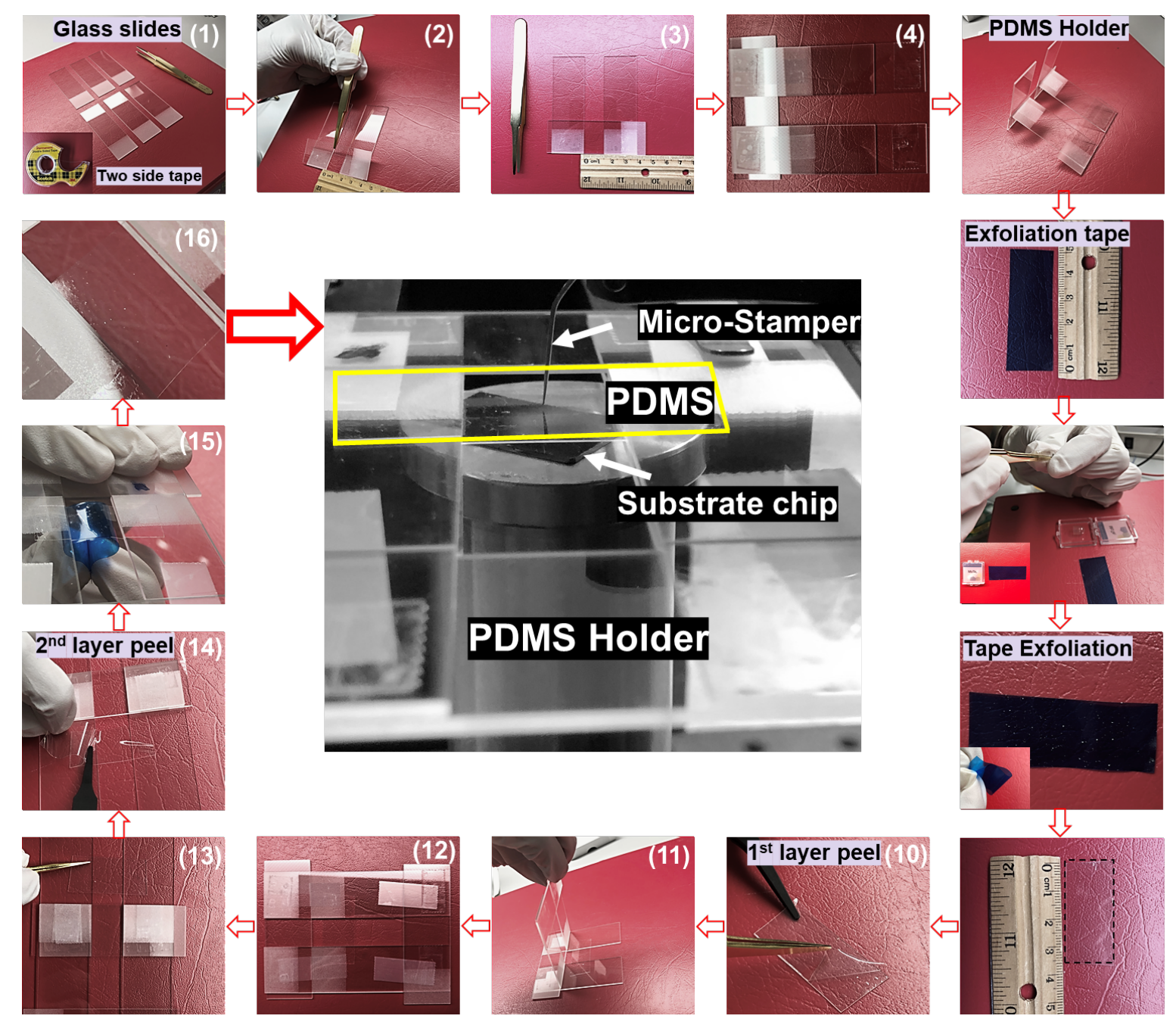

Figure 4. Step-by-step pictorial flowchart for PDMS holder preparation and modified exfoliation technique. The holder can be reused once prepared by replacing the PDMS film clamped in between the glass slide frame. The preparation of the holder requires thin glass slides $(\sim 1.2 \mathrm{~mm})$, Gel-Pak thin PDMS film 
(17 mils), double-sided and single-sided adhesive tapes, pair of tweezers, weak adhesion exfoliation tape, ruler, and 2D material crystal. The figure in the center shows the placement of the holder on the stage for finding and positioning the $2 \mathrm{D}$ material flakes

The tape is usually then contacted with the substrate/target to transfer the flakes that have adhered to it. However, residues from the tape glue and other particles stuck to the tape are also added, resulting in a decreased yield of good-quality flakes. In addition, this spreads a lot of clusters of overlapping flakes all over the substrate, resulting in cross-contamination. The tape is gently pressed against the suspended PDMS film on the holder a few times. This permits the loosely bonded flakes on tape to adhere to the PDMS, reducing flakes clumping and allowing for more isolated flakes to be transferred. A complete pictorial procedure can be seen in Fig. 4.

\section{d) Transfer Process}

The transfer process is simple and highly effective using the 2DMTS (Fig. 5). The entire system is independent or requires minimal need for user experience and training. This makes this system more user-friendly as compared to other systems and avoids long training periods for a new user. The entire transfer process can be implemented in three simple steps: (i) Finding the flakes (ii) Positioning the flake and target area (iii) transferring the material. The desired size and shape of the flake can be selected on the PDMS by moving in the $\mathrm{XY}$ direction and using the measurement feature in the camera software. The microscope
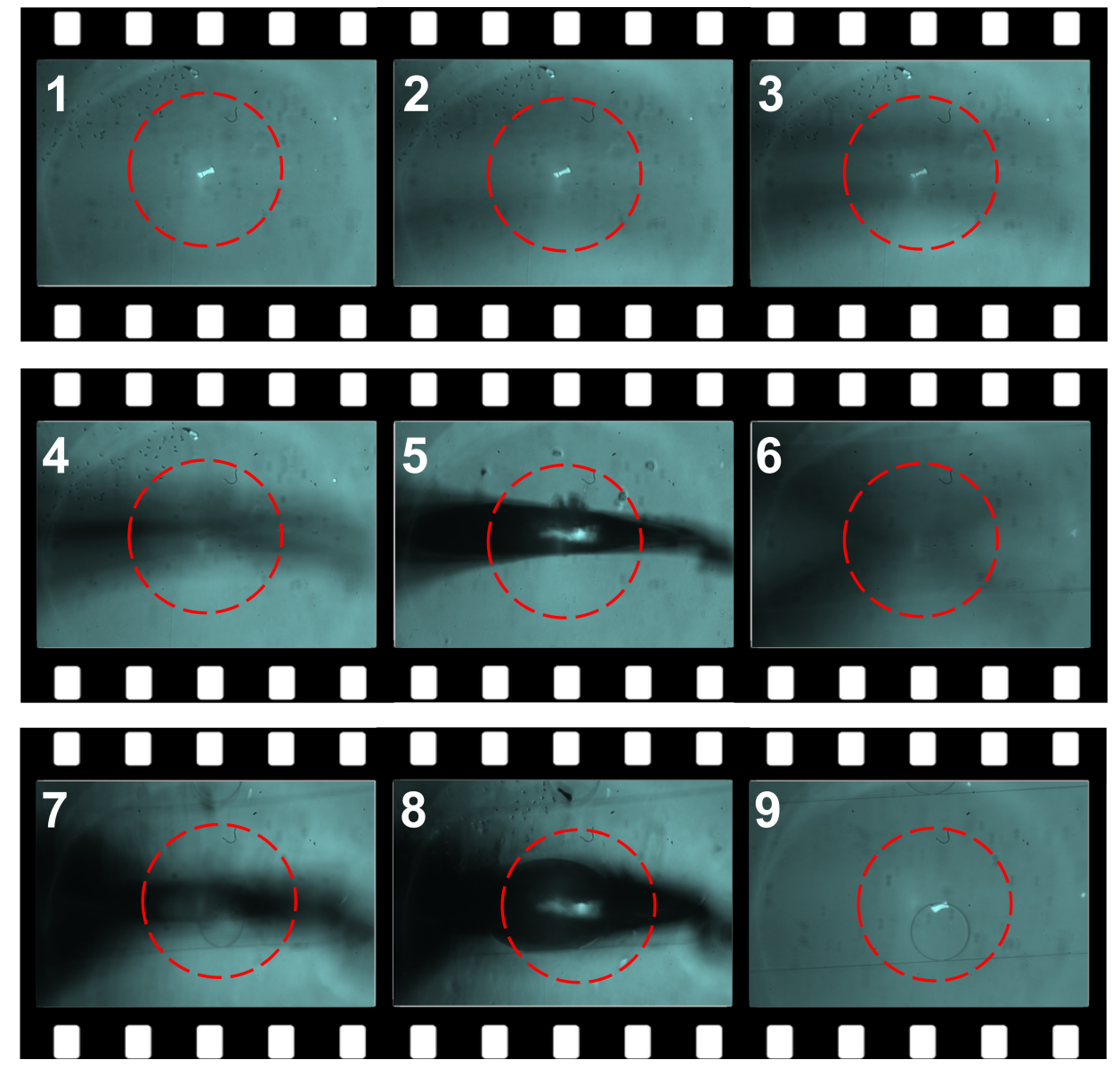

Figure 5. The real-time video frames were acquired during the transfer of $\mathrm{WSe}_{2}$ flakes onto a silicon Microring resonator structure. The numbers on each frame denote the order in which the frames were 
captured during the transfer. Frames \#1 - \#4 show the micro stamper approaching the PDMS. Frame \#5 shows the micro stamper tip contacting the PDMS, which continues to advance towards the target, while frames \#6 \#9 show the stamper reverting to its original location.

focal adjustment can be used to transfer the focus of vision to the micro-stamper, PDMS, and through the PDMS on target, once the PDMS holder is loaded on the stage. Using the micro-manipulating stages, a software crosshair assists in positioning the 2D material flake, target, and needle to a precise overlapping position. Following this alignment, the target and PDMS can be adjusted in the $\mathrm{XY}$ direction as needed to locate the flake and/or adjust the target's position. Once aligned to the center of the crosshair (homing position), the microstamper can be moved in the $Z$ direction to make transfers. When PDMS touches the sample, the live video exhibits distinct indicators, allowing the operator to avoid over pressing the micro-stamper on the material while transferring. Once the PDMS has contacted the substrate, the operator can carefully bring the micro-stamper back to the homing position and repeat the operation for other flakes on the PDMS and target locations on the substrate. The micro stamper exerts stress of $\sim 450 \mathrm{kPa}$ on the substrate and 2D flake while transferring simulated by ANSYS static structural simulation. This pressure is experimentally measured by placing a pressure sensor under the target substrate resulting in 13kPa which is within the elastic limit of 2D material (GPa) [30], [31], and PDMS material (MPa) [32]. The simulation results for the stress and strain analysis of the 2DMTS on PDMS and substrate are discussed in detail in the supplementary section (Fig. S1).

\section{e) Nano-fabrication process}

The devices discussed in the application section in this article follow similar process steps unless otherwise specified further in the discussion. The study is performed on commercial Si photonic chips (Applied Nanotools INC.) tape-out. The flakes in all the applications discussed were transferred using the proposed system. The samples were rinsed clean using acetone followed by isopropyl alcohol for few minutes, both, before and after transfer. The electrical contacts were patterned using an e-beam lithography process assisted by pre-patterned alignment markers for positioning the flakes while making contacts. The Au/Ti $(45 \mathrm{~nm} / 5 \mathrm{~nm})$ metal deposition was performed by e-beam evaporation technique followed by a lift-off process using acetone at room temperature.

\section{f) Electrical and optical measurement setup}

The experimental setup for measuring the heterogeneously integrated TMDC-Si devices comprises a tunable laser source (Agilent 8164B) and a broadband source (AEDFAPA-30-B-FA) from where light is coupled into the grating coupler optimized for the propagation in the waveguide for $1550 \mathrm{~nm}$ wavelength. The light output from the Si-MRR is coupled to the output fiber via a grating coupler and detected by a detector or an optical spectral analyzer (OSA202). A source meter (Keithly 2600B) was used for electrical measurements. A tunable (NKT SUPERCONTINUUM Compact) source and fixed wavelength laser diode module (CPS980 Thorlbas, INC.) at 980nm wavelength was used as sources for measuring the heterojunction based PN junction photodetector devices.

\section{Applications}

\section{(a) Electrical Characterization of 2D materials post-2DMTS Transfer (Fig. 6)}

An extensive study has been performed on electrical properties of $2 \mathrm{D}$ materials, indicating the effect of impurities, trapped contaminants, and defects caused while transferring the material or post-process effects [33]-[35]. It is important to study the 
electrical properties of the material after contact formation for optimized performance as studied in [36]-[45]. However, achieving consistent results in the electrical properties of such devices is challenging for other transfer methods discussed earlier. To demonstrate consistency in the quality of flakes transferred using the proposed system we performed transmission line measurement on these flakes for sheet resistance measurement. The results were normalized by the area of the flake to remove the effect of the shape and size of flakes from the resistance measurement. Flakes with uniform thickness surfaces were considered for measurement confirmed by Raman spectroscopy before making the contact pads. As seen in the microscope image in Fig. 6a inset, $\mathrm{MoS}_{2}$ flakes were transferred on $\mathrm{SiO}_{2}$ substrate, and contacts were formed for different channels lengths from $0.5 \mu \mathrm{m}$ to $3.5 \mu \mathrm{m}$ with a step size of $0.5 \mu \mathrm{m}$. This type of electrical characterization helps in designing the next-generation devices for improved optoelectronic device performances [46], [47]. Fig. 6a shows the variation in sheet resistance with varying channel length on the same flake representing the sheet resistance. Several such devices were fabricated at once by transferring multiple flakes on $\mathrm{SiO}_{2}$ substrate for testing repeatability in achieving similar electrical properties. Repeatable electrical characterization was achieved by building 18 devices and observing their respective contact resistance $\left(R_{c} \sim<50 \mathrm{k} \Omega\right.$ ) and sheet resistance $\left(R_{S} \sim<10 \mathrm{k} \Omega / \mu \mathrm{m}\right)$ of $\mathrm{MoS}_{2}$ flake as seen in Fig. $\mathbf{6 b}$. The electrical characterization of $2 \mathrm{D}$ materials is an important aspect to be studied for building electro-optic devices, magnetostatic devices, and stretchable electronic devices [47]-[50].

a

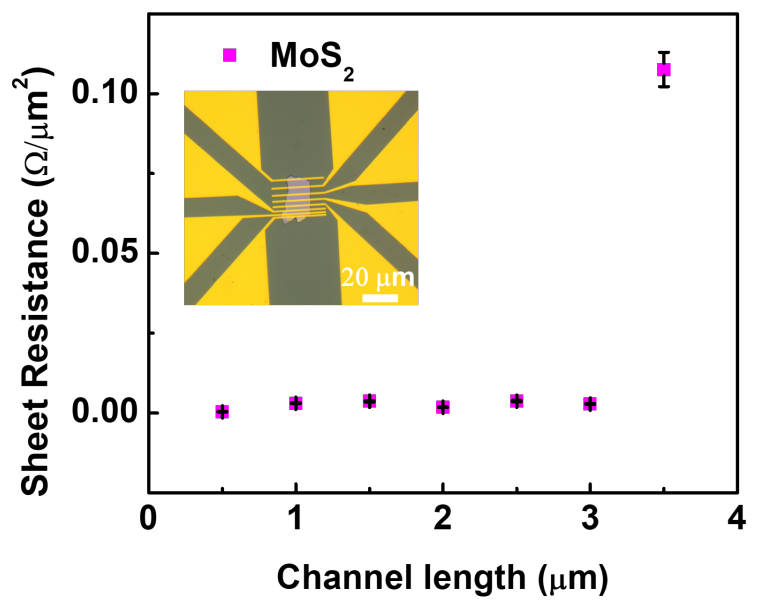

b

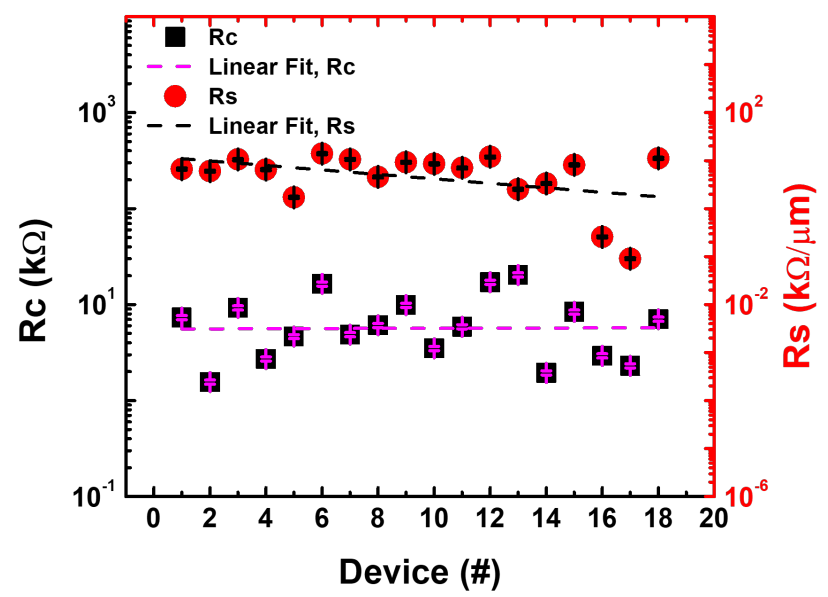

Figure 6. Electronic resistivity test of 2D flakes transferred with 2DMTS. (a) 2-terminal resistance measurement of individual $\mathrm{MoS}_{2}$ flakes represented in the microscope image was performed to determine channel length-based sheet resistance of the material for electrical characterization. A microscope image of the device in the inset of $\mathrm{MoS}_{2}$ flake transferred on $\mathrm{SiO}_{2}$ substrate after designing the contacts for various channel lengths. (b) Comparative study for achieving repeatable electrical properties can be observed in the form of contact resistance $\left(\mathrm{R}_{\mathrm{c}} \sim<50 \mathrm{k} \Omega\right)$ and sheet resistance $\left(\mathrm{R}_{\mathrm{s}} \sim<10 \mathrm{k} \Omega / \mu \mathrm{m}\right)$ for 18 devices.

\section{(b) Precise Transfer on an Array of Devices On-chip (Fig. 7)}

It is important to increase the yield of devices that can be fabricated for extensive research studies or commercial mass fabrication. A simple experiment is demonstrated here which proves the precise and accurate transfer capability of the system producing multiple devices on a chip in less than one minute spent on each device to transfer and few minutes in searching the desired flakes. The flake search time of few minutes can be minimized by prescanning the PDMS film and registering the flake positions using the top-mounted 
camera and a computer program by image processing. A commercial Si-photonic chip with arrays of micro-ring resonator (MRR) devices separated by $200 \mu \mathrm{m}$ distance was used in this experimentation. Each MRR was positioned under the system to transfer one flake in a sequence without changing any settings of the setup. The 2D material flakes, here $\mathrm{MoS}_{2}$, were chosen at random and were targeted to be transferred at the same position on MRR for each device. Such transfers were made on 15 devices in a column on-chip of which three devices are shown in Fig. 7a with a close view for the transferred flake in Fig. $\mathbf{7 b}$. Such robustness of the setup can be used to make devices for producing active and passive tunable devices [9], [51]-[53] with higher yield and repeatable results as discussed further.

\section{(c) Post passive tunning of Silicon}

The designed performance of silicon photonic circuits may vary due to fabrication uncertainties or incorporation of multiple post-fabrication process steps for fabricating active tunning devices on-chip. Also, tunning photonic circuits with precise control over the amount of tunning capability can be used for applications in neuromorphic computing [51], [52], [54][58]. A novel study was extensively discussed by [4] in past showing tunning of silicon microring resonator (MRR) coupling efficiency from under-coupled to the over-couple regime after the MRR was fabricated using $\mathrm{MoS}_{2}$ using such stamping technique. Such studies can be crucial in understanding the effect of different 2D materials on optical properties after integration. Further helping to understand the optical properties of 2D materials like refractive index, optical absorption, phase change, and emission. We the proposed system such devices and study can be accelerated for studying any $2 \mathrm{D}$ material characterization study.

We here demonstrate a similar device as discussed in [4], showing enhanced coupling efficiency of Si-MRR from under coupled regime to critical coupling regime as seen in Fig. 7c. The MRR was transferred with two different small flakes as discussed in Fig. 7 demonstrating precision in transferring flakes on devices extremely close to each other (here 25um), as seen in Fig. 7d. The MRR spectral resonance enhancement was observed after transferring each of the two flakes to understand the change in coupling conditions by tunning the effective refractive index given by $(\Delta n)_{(\text {eff,ring })}=\left((2 \pi R-L)^{*} n_{(\text {eff,bare })}+L^{*} n_{\text {eff }}\right) / 2 \pi R$ and seen in Fig. 7e [4]. Optical loss due to scattering from material edges and change in the imaginary part of the effective refractive index of the MRR was in total observed to be about $0.00251 \mathrm{~dB} / \mu \mathrm{m}$, represented in Fig. 7f. 
a

b

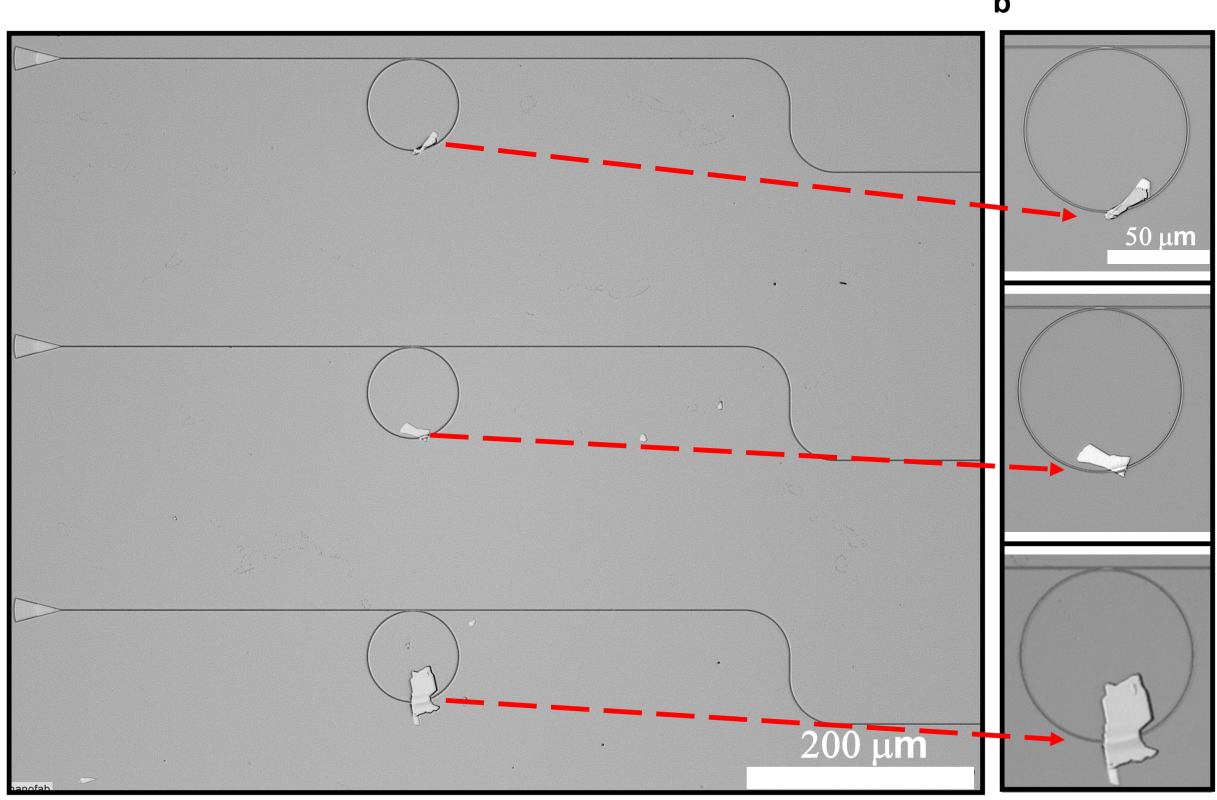

c

d
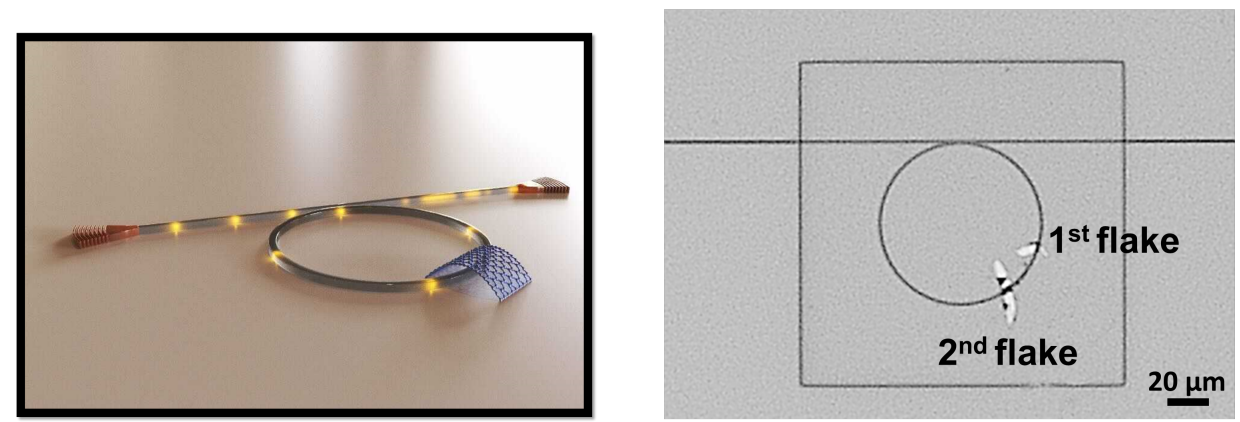

e

f
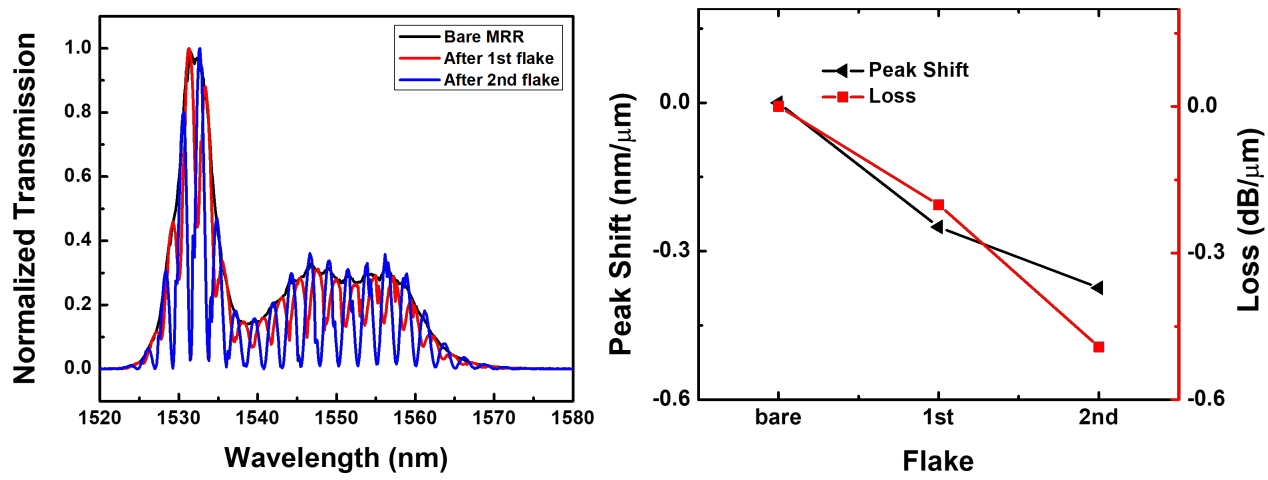

Figure 7. 2DMTS performance test for accuracy, precision, and repeatability on assembling 2D structures atop photonic integrated circuit waveguides. (a) Optical microscope images at 20x magnification of $\mathrm{MoS}_{2}$ flakes transferred on Si-MRR devices in an array on-chip separated by $200 \mathrm{~nm}$ distance from each other. (b) Zoomed in at 50x magnification to show the flake position on devices. Silicon micro-ring resonator post coupling enhancement using $\mathrm{MoS}_{2}$ thin flakes. (c) Schematic (d) optical microscope image of an MRR ( $D=80 \mu \mathrm{m} \& \mathrm{~W}=500 \mathrm{~nm}$ ) covered by two $\mathrm{MoS}_{2}$ flakes precisely transferred using our developed 2D printer technique. (e) Spectral transmission output before and after the transfer of $\mathrm{MoS}_{2}$ showing improvement of coupling efficiency. The improved resonance fringes can be observed in the graph (f) Resonance peak shift and optical loss after transfer of each flake can be observed showing linear absorption loss and variation in peak shift of resonance. 


\section{(d) Strain Engineered $\mathrm{MoTe}_{2}$ Integrated Photodetector on Silicon microring resonator}

(MRR)

We demonstrate a heterogeneous integration of 2D material using the proposed transfer system on a silicon photonic circuit on-chip using a few-layer $\mathrm{MoTe}_{2}$ flake. Owing to the bandgap of $\mathrm{MoTe}_{2}$, the optical absorption of the material is not suitable for $1550 \mathrm{~nm}$ light detection. Using the proposed 2DMTS, the material can be strained on Si waveguides which enables bandgap tuning of $\mathrm{MoTe}_{2}$. Therefore, allows the material to absorb at

a

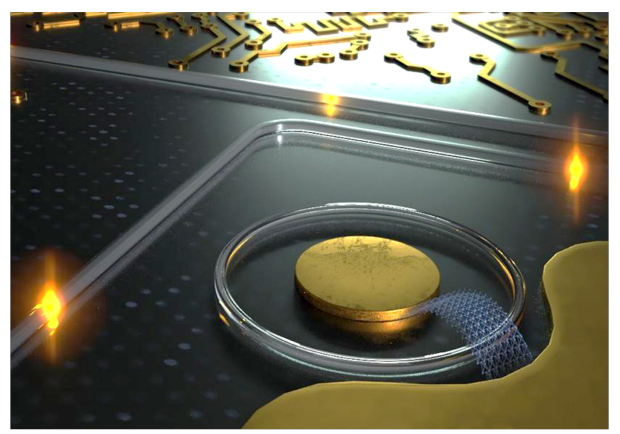

C
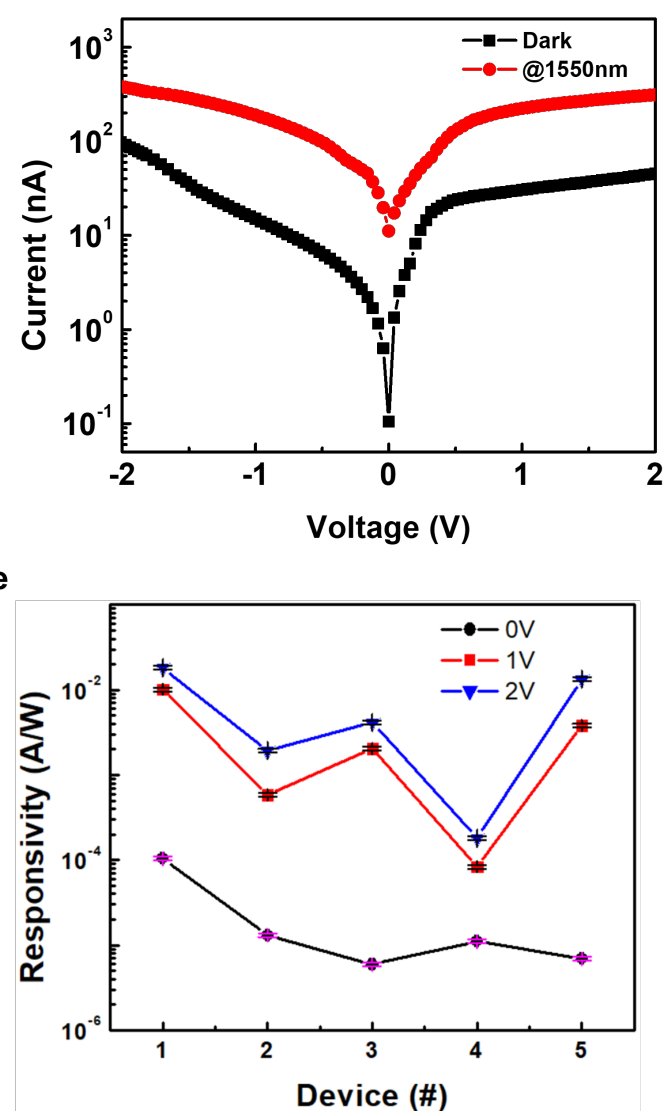

b

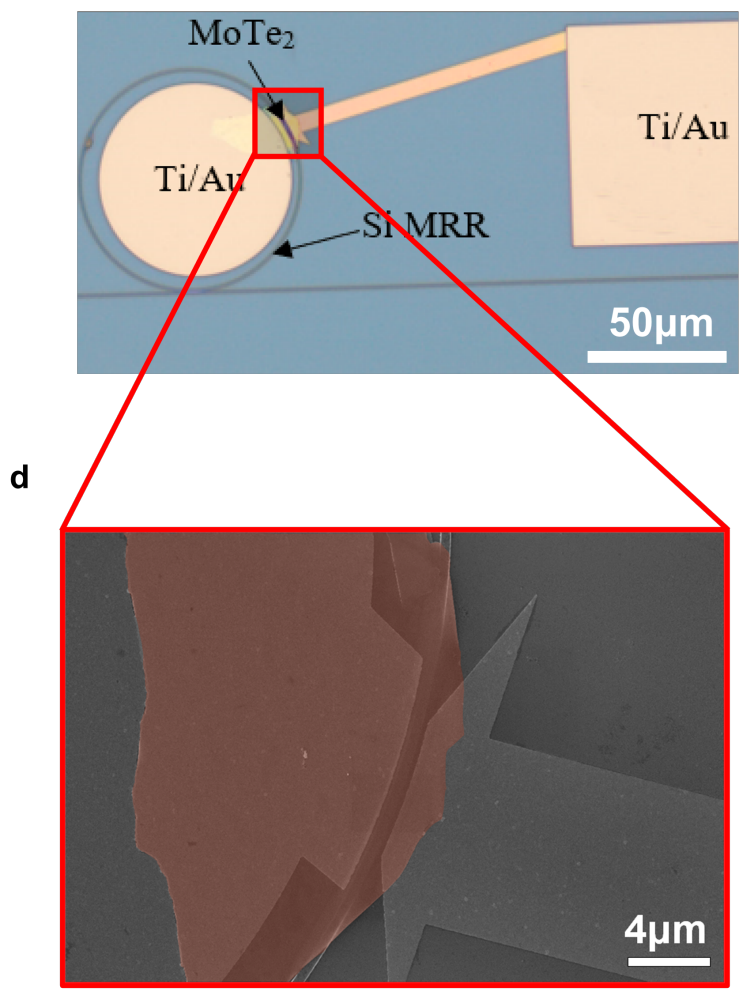

$\mathbf{f}$

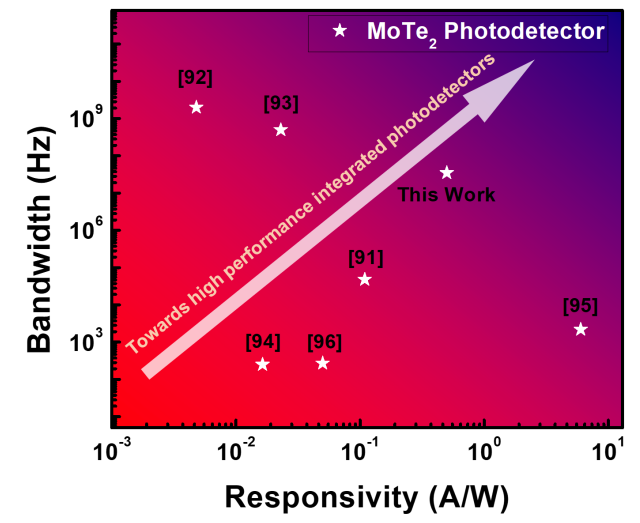

Figure 8. A Si-MRR integrated strain engineered MoTe 2 photodetector. a) Schematic representation of $\mathrm{MoTe}_{2}$ based integrated photodetector for strainoptronics application built using the 2DMTS b) The structure of Si-MRR (radius $(r)=40 \mu \mathrm{m}$, height $(h)=220 \mathrm{~nm}$, width $(w)=500 \mathrm{~nm}$ ), and electrical contacts are represented in the optical microscope image. c) The I-V characteristics show the enhancement in current under the illumination of light coupled through Si-MRR at 1550nm wavelength. d) SEM image shows the coverage of 
flake on Si-MRR showing the coverage of the flake on the device e) Performance of five different photodetector devices represented by responsivity measured at zero bias (0V), $1 \mathrm{~V}$, and $2 \mathrm{~V}$ showing enhancement in magnitude due to improvement in photogenerated carrier collection under influence of bias. f) Various photodetector devices discussed in [8], [59]-[64] are tabulated for understanding the bandwidth performance of $\mathrm{MoTe}_{2}$ based photodetectors

lowered bandgap due to induced strain. A single few-layer MoTe $\mathrm{e}_{2}$ flake was transferred on a silicon MRR based photodetector with $0.5 \mathrm{~A} / \mathrm{W}$ responsivity operating at $1550 \mathrm{~nm}$ wavelength as discussed in [8]. A similar device has been designed and fabricated here for demonstrating the capability of the transfer system for realizing and studying such devices. The flake was transferred on a silicon MRR precisely using a micro-stamper and was patterned using the lithography process for contacts as seen in Fig. 8a. The microscope image as seen in Fig. $\mathbf{8 b}$ shows the device structure with electrical contacts. The currentvoltage (I-V) characteristics of the device can be observed in Fig. 8c. A high responsivity of $0.02 \mathrm{~A} / \mathrm{W}\left(\mathrm{R}=I_{\text {photon- }} I_{\text {dark }} / \mathrm{P}_{\text {in }}\right)$ was achieved for $2 \mathrm{~V}$ electrical bias at $1550 \mathrm{~nm}$ telecommunication wavelength. The dark current is observed around 50nA. A close view of the SEM image of the flake placed on the MRR can be seen in Fig. 8d. Multiple photodetector devices were studied here for different thicknesses, shapes, and sizes of the flakes, on the same chip with each MRR away from each other by 200 um showing the capability of array transfer represent in Fig. 1b to develop such devices with high repeatability. The responsivity of five such photodetectors at no bias (0V), $1 \mathrm{~V}$, and $2 \mathrm{~V}$ can be seen in Fig. 8e integrated on Si-MRR with light coupled at $1550 \mathrm{~nm}$. The variation in performance for each device is due to variation in the coverage area of flake due to different sizes and variations in thickness. A detailed study of the effect of strain used to tune the absorption bandgap of $\mathrm{MoTe}_{2}$ for such devices is discussed in [8] towards strainoptronics applications. These types of devices are promising for future on-chip detection and modulation of the signal without a complex fabrication process and high cost of production [6], [48], [65].

\section{(e) Strain effect on WSe 2 bandgap stretched on nanostructures}

The atomically stacked 2D materials with each atomically thin layer adhered to each other under vDW forces show unique and strong tunning capabilities of modulating its properties by using mechanical forces. The properties of $\mathrm{WSe}_{2}$ like photoluminescence(PL) enhancement, bandgap tuning, quantum emission, and optical absorption can be modulated by generating strain in the material [66]-[69]. Here we demonstrate the strain effect on $\mathrm{WSe}_{2}$ multilayer using triangular geometric pillars. The pillars are etched from a SiN substrate of height $220 \mathrm{~nm}$. The structures are equally spaced to allow part of the flake to slack in between pillars and also stay suspended in few parts as seen in Fig. 9a. Fig. 9b(I), (II) show the microscope image of the nanopillars after the etching process and after a large $\mathrm{WSe}_{2}$ flake transfer on the same area using the proposed transfer system respectively. In this configuration, it was possible to suspend the flake like a membrane on multiple nanopillars producing a high strain gradient along the pillar area. The corners of the triangular area help in strongly straining the membrane due to the virtue of the geometric shape. To understand the effect of induced strain, here, we performed photoluminescence and Raman spectroscopy spatial mapping. The Raman active modes of $\mathrm{WSe}_{2}$ are represented in Fig. 9c. The PL spectra were analyzed by fitting the data to find the significant peak positions as seen in Fig.9d. The peak positions are highlighted in the figure at which the PL map analysis is performed for strain effects. 


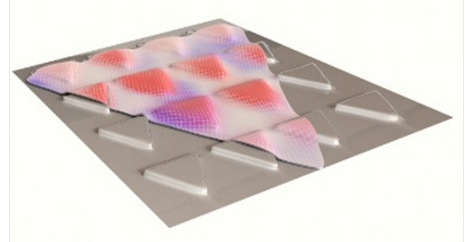

C

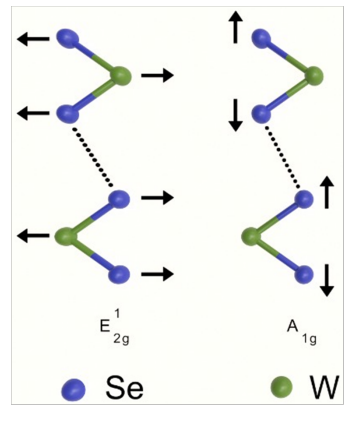

b

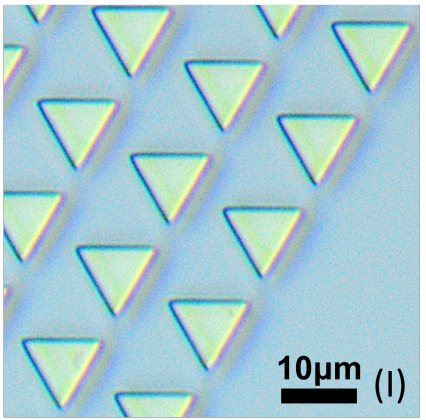

d

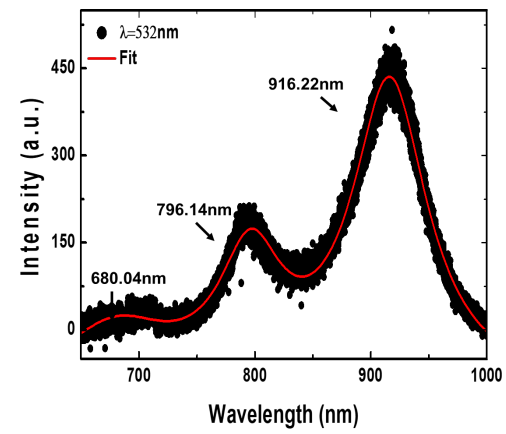

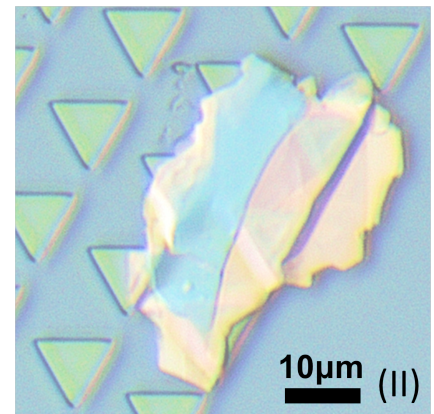

e

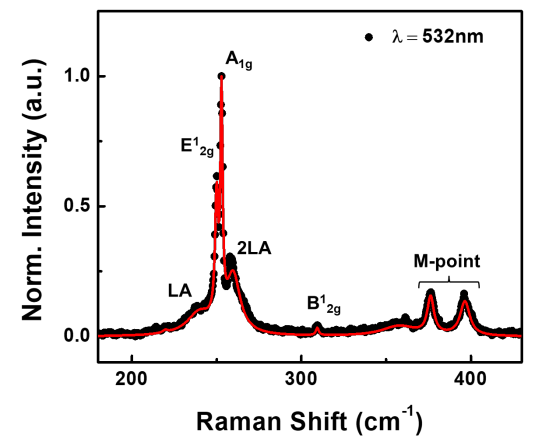

f
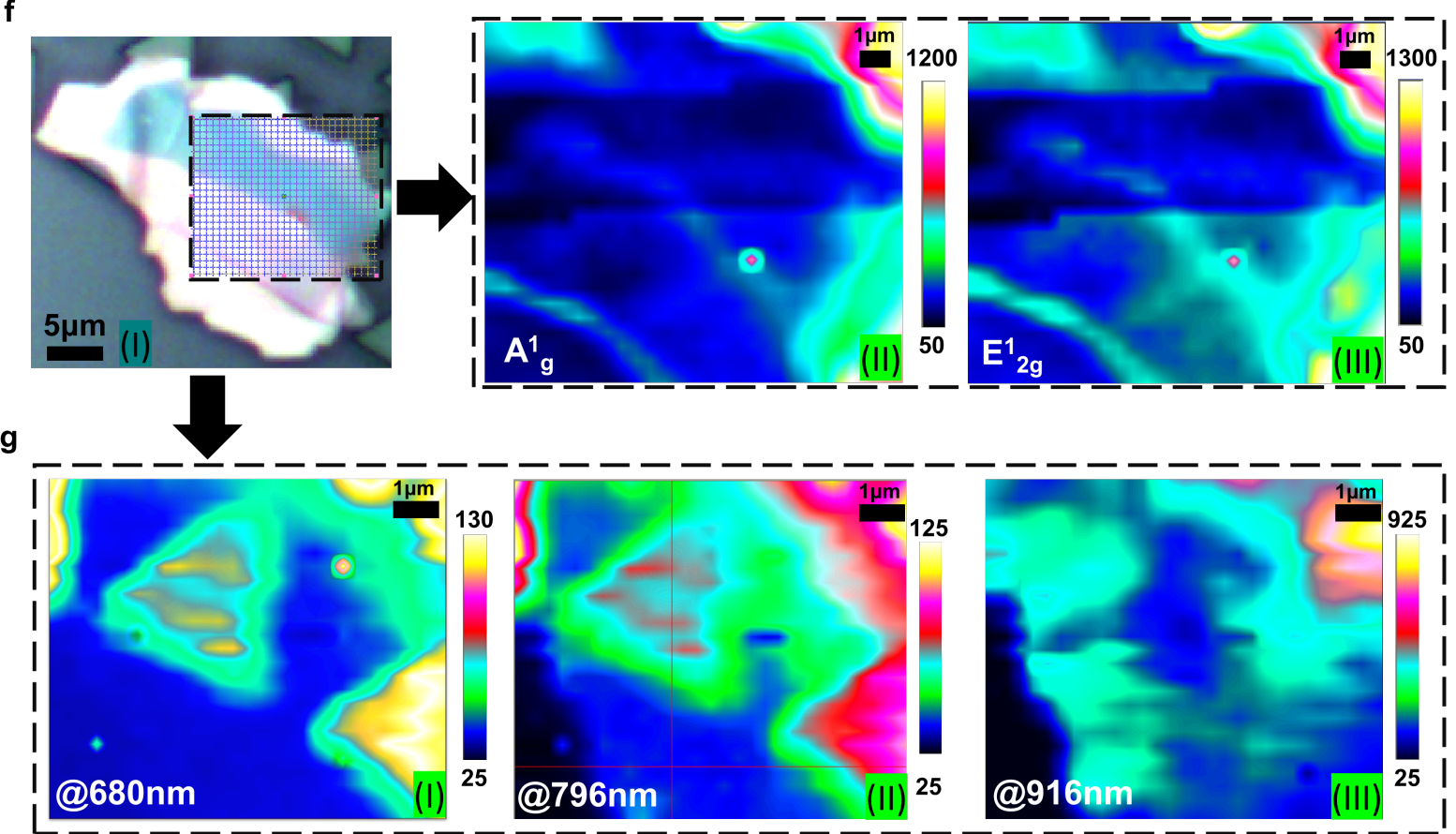

Figure 9. Strain-induced in multilayer $\mathbf{W S e}_{2}$ using triangular nanopillars. (a) Schematic representation of strained multilayer $\mathrm{WSe}_{2}$ membrane on triangular nanopillars. (b) Optical microscope images for (I) SiN triangular nanopillar structures (II) WSe $\mathrm{WS}_{2}$ membrane (after transfer) using the 2D printer setup on the nanopillars. (c) Schematic representation of $E^{1}{ }_{2 g}$ and $A_{1 g}$ Raman active modes of WSe $e_{2}$ (d) PL spectra of $W_{S} e_{2}$ membrane showing the fitted curve highlighting the significant active peaks. (e) Raman spectra showing the active modes $\left(\lambda_{\text {excitation }}=532 \mathrm{~nm}\right)$. (f) Optical image of mapping area highlighted with the grid selection for Raman and PL mapping points (I), Raman intensity map for the strained area of the WSe membrane at $\mathrm{A}_{1 \mathrm{~g}}$ mode (II), and $E^{1}{ }_{2 g}$ mode (III). (g) PL mapping realized at significant PL peaks for WSe 2 at $680 \mathrm{~nm}, 796 \mathrm{~nm}$, and $916 \mathrm{~nm}$ showing intensity enhancement for $680 \mathrm{~nm}$ and $796 \mathrm{~nm}$ peak position while a reduction in intensity at 916nm peak position. 
The crystal lattice vibrations of bulk $W S_{2}$ include Raman active modes of $A_{1 g}, E_{1 g}$, and $E_{2 g}$ [70] (Fig.9e). In Raman tensor analysis, the E-modes and A-modes are studied using circularly polarized helicity configuration [71]. In LR configuration (incident with left circular polarization and collect right circular polarization signal), we can get $247.4 \mathrm{~cm}-1$ peaks consistently which denotes $\mathrm{E}^{1}{ }_{2 \mathrm{~g}}$ mode peak, while in LL configuration (incident with left circular polarization and collect left circular polarization signal), we have $249.5 \mathrm{~cm}-1$ peak which corresponds with $A_{1 g}$ mode (Fig. 9e). As seen in Fig. 9f(I), an area was selected on top of few pillars with a small grid representing the mapping density using during the measurements. The effect of strain caused physical atomic arrangement variation in the crystal leading to change in vibrational modes of the material [66], [69]. Fig. 9f shows the Raman mapping profile of the $\mathrm{WSe}_{2}$ membrane strained on a pillar showing the change of almost three times than unstrained region in the intensity of $A_{1 g}$ mode (II) and $E_{2 g}^{1}$ mode (III). The intensity variation here also shows the curved surface profile of the membrane on the pillars and the change in the peak intensity due to the strain introduced in the material. Further to test the optical response of the material under strained conditions, photoluminescence spectroscopy was performed to observe intensity enhancement at the significant peaks observed in Fig. 9d. For WSe $\mathrm{W}_{2}$, the PL spectra mapping was performed at $680 \mathrm{~nm}, 796 \mathrm{~nm}$, and $916 \mathrm{~nm}$ to understand the effect of strain on

each of them. Such studies can help in understanding the material properties to design and fabricate devices for exotic applications in various fields. The intensity map of PL for the strained region on one of the pillars at each of the significant peaks can be seen in Fig. $\mathbf{9 g}$. An enhancement of about 4.5x and 3x was observed at $680 \mathrm{~nm}$ (I) and $796 \mathrm{~nm}$ (II) peak positions respectively. But a reduction in the intensity of about $2.7 \mathrm{x}$ is observed at $916 \mathrm{~nm}$ (III). The selective tuning of the photoluminescence response of $\mathrm{WSe}_{2}$ can is used for designing optical filters or spectral selective absorbers integrated on photonic circuits [72][74].

\section{(f) Building 2D material Heterostructures}

When we assemble numerous 2D crystals into a vertical stack, a multitude of possibilities emerge for exploring various properties exhibited by these materials. Such heterostructures, held together by vdW forces, allow significantly more combinations than any other standard material stacking growth process. The intricacy of the heterostructures that could be constructed with atomic accuracy is increasing as the family of $2 \mathrm{D}$ crystals grows [75]-[80]. Such 2D heterostructures can be used to build devices for electronic or electro-optic applications, superlattices, and quantum applications [50], [73], [77], [79], [81][96]. Here, we demonstrate a small example of building a $\mathrm{MoS}_{2} / \mathrm{WSe}_{2}$ heterojunction using the proposed 2D transfer system. A simple demonstration of combining individual unique properties of $\mathrm{MoS}_{2}$ and WSe 2 by forming a junction from heterostructure. Both the $\mathrm{MoS}_{2}$ and

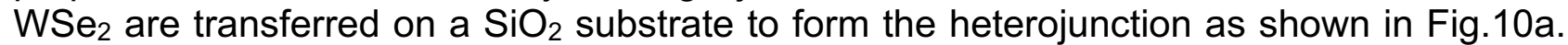
The inset in Fig. 10a shows the dark field microscope image of the heterostructure confirming the clean surface on the flakes and substrate. By experimental analysis of the unique material signature exhibited by Raman spectroscopy, we observe the combined effect of both the materials in a heterostructure. As seen in Fig. 10b, the Raman spectra for individual flakes of $\mathrm{MoS}_{2}$ and $\mathrm{WSe}_{2}$ can be observed with their active mode peaks. The $\mathrm{MoS}_{2} / \mathrm{WSe}_{2}$ heterojunction Raman spectra exhibit the Raman active modes of both the materials combined. The location for detecting the Raman signal is marked on the optical 
image in Fig. 10a. The data were corrected with background signal and baseline correction for removing the unwanted noise in the signal. This example shows the capability of the setup to realize heterostructure formation for different applications. The angle between each layered stack can be controlled in the setup for twistronics and superlattices applications as well but not discussed here.

a

b
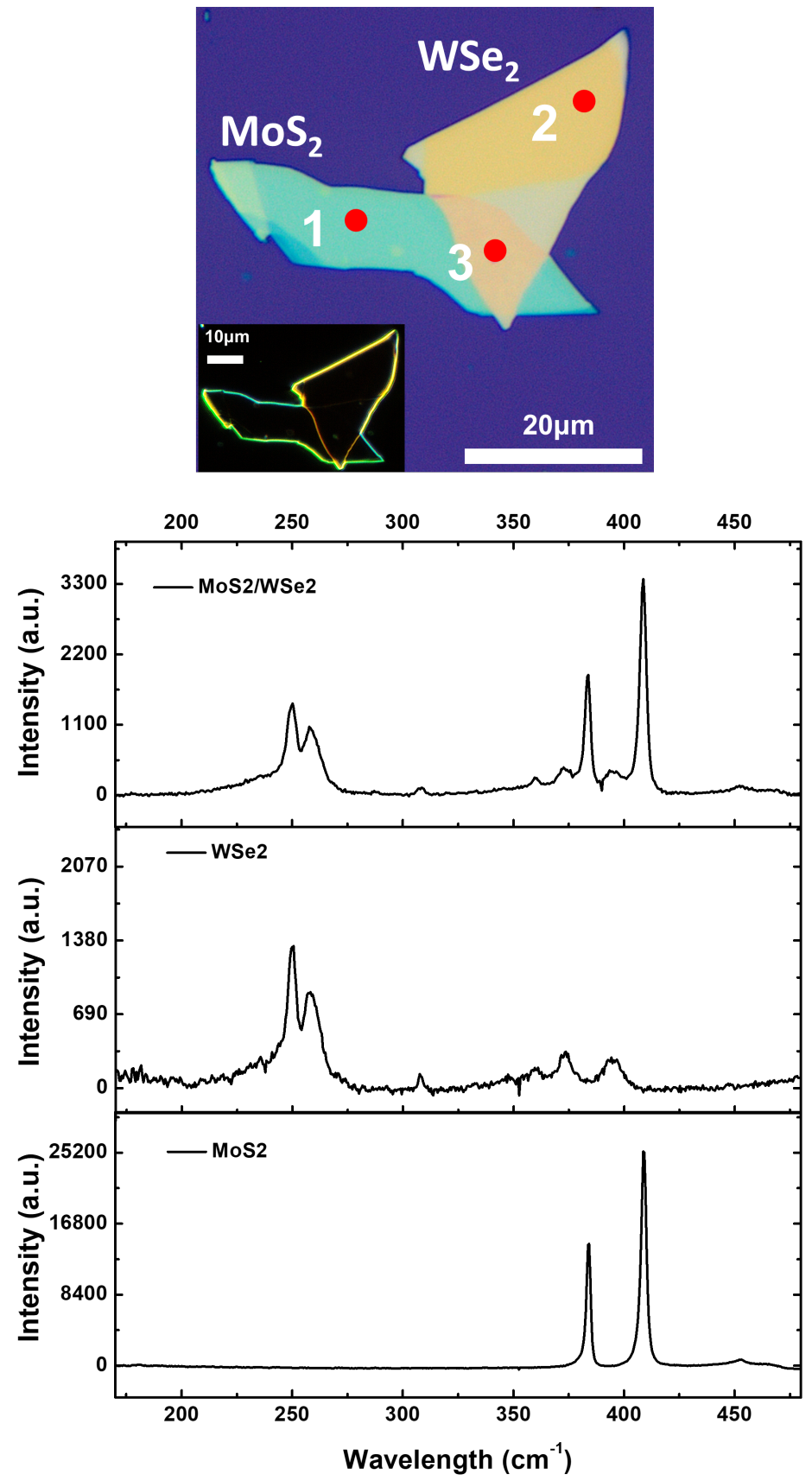

Figure 10. $\mathbf{M o S}_{2}$ and $\mathbf{W S e}_{2}$ multilayer heterostructure. (a) Optical microscope image of $\mathrm{MoS}_{2} / \mathrm{WSe}_{2}$ heterostructure built on $\mathrm{SiO}_{2}$ substrate. The inset shows the dark field microscopy image of the heterostructure indicating no residues on the surface of the materials and substrate. (b) Raman spectra for $\mathrm{MoS}_{2}, \mathrm{WSe}_{2}$, and $\mathrm{MoS}_{2} / \mathrm{WSe} \mathrm{e}_{2}$ heterostructure showing the combined Raman signature of $\mathrm{MoS}_{2}$ and WSe $\mathrm{W}_{2}$ at the junction formed by them. 


\section{(g) InSe based PN-Junction Heterostructure Photodetector}

Since past decades, 2D materials have been studied as promising photodetector materials, by changing the layer numbers or forming vdW heterostructures, owing to their high responsivity, fast response, broadband detection, low dark-current, and photodetectivity [35], [65], [95], [97]-[107]. The operation of these high-performance devices demands high bias voltage leading to large power consumption. This limits technological applications in extreme environments, biomedical imaging, portable devices, etc. 2D indium selenide (InSe) has recently been investigated showing higher ultrasensitive photodetection characteristics [108] than other 2D semiconducting materials such as $\mathrm{MoS}_{2}$ and $\mathrm{WSe}_{2}$ [108][114]. Junction-based, e.g. $p$ - and $n$ - doped materials enabled realization in heterostructure devices by the formation of an atomically sharp p-n junction [94], [109], [115].

a

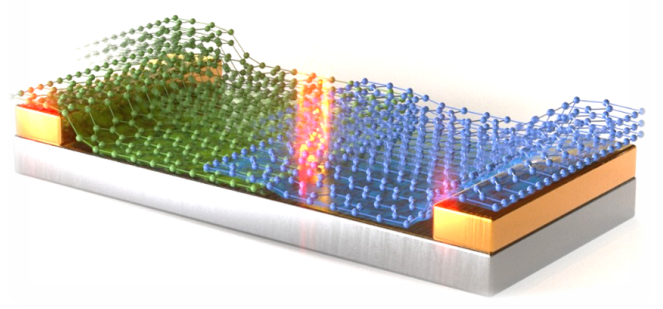

C

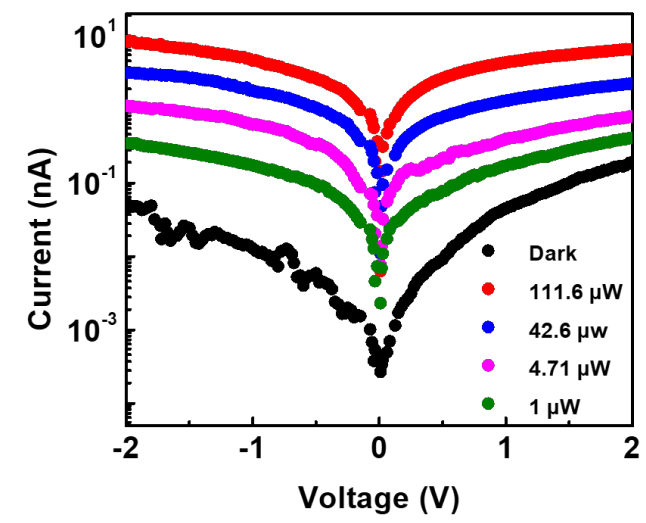

b

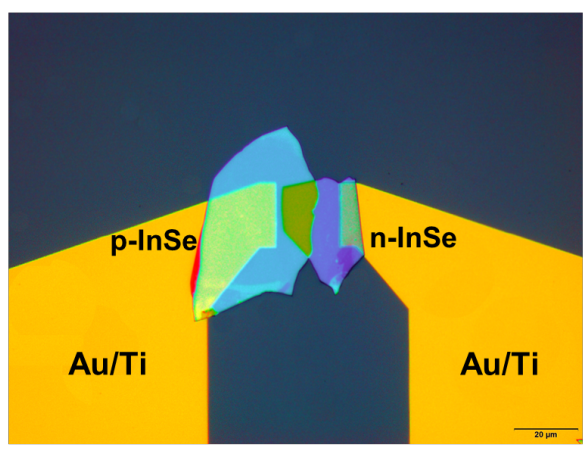

d

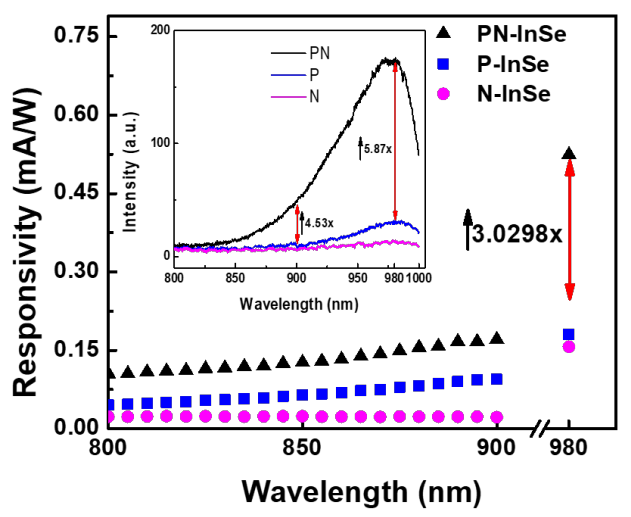

Figure 11. 2D pn-junction heterostructure-based photodetector using 2DMTS. (a) Schematic representation of the pn junction device. (b) Optical microscope image for 2D heterostructure stack of $p$ - and n-doped InSe flake on $\mathrm{SiO}_{2}$ substrate transferred on Au/Ti metal electrodes using the transfer system. (c) I-V characteristics of the device were tested at different optical power illumination at 980nm for photocurrent mapping. (d) The spectral responsivity of the pn junction device compared with control sample devices with $n$ and p-doped channel photodetectors showing enhancement of about 3.0298x. The inset shows the PL emission spectra intensity enhancement at the $p$ junction area compared to $p$ and $n$ regions.

Using 2DMTS, here we demonstrate a vDW heterostructure-based photodetector for near-infrared (NIR) spectrum absorption capable of efficient photo-detection operation at zero-bias enabled by a built-in voltage from the 2D material PN junction [116]. The important aspect of vDW 2D heterostructure is achieving a clean interface between the layers. In general, the quality of realizable heterojunctions is affected by chemical or mechanical 
degradation due to the presence of residues from adhesive from Nitto tape, polymer adhesives, or wet chemicals depending on the transfer method. By using a dry transfer medium and the mechanical stamping mechanism of 2DMTS this issue is eradicated. We heterogeneously integrated $\mathrm{p}-(\mathrm{Zn})$ and $n-(\mathrm{Sn})$ doped InSe material using 2DMTS on prefabricated electrical contacts ( $\mathrm{Au} / \mathrm{Ti})$ (Fig. 11a). The sample was kept in the vacuum chamber for 30 minutes for better layer-layer and layer-metal adhesion. The optical microscope image seen in Fig. 11b shows the actual device configuration and structure of the electrical contacts. The center region forms the $p-n$ junction formed by the heterostructure. The device's current-voltage characteristics were tested to observe dark current and photocurrent generation for $980 \mathrm{~nm}$ wavelength illumination. The change in photocurrent for different optical power was also tested at $980 \mathrm{~nm}$ showing a gradual increase in photocurrent as seen in Fig.11c. the spectral response of the pn junction photodetector was performed for a range of wavelengths from $800 \mathrm{~nm}$ to $900 \mathrm{~nm}$ using a tunable broadband source and also at 980nm using a laser module (VCSEL). A similar device with only $n$-doped and $p$-doped InSe channels was fabricated for comparison with the pn junction configuration of the device for the spectral response as seen in Fig. 11d. An enhancement of about $3 x$ was observed in responsivity of about $0.524 \mathrm{~mA} / \mathrm{W}$ for the pnjunction InSe photodetector as compared to only p- or n-doped InSe photodetector. The responsivity enhancement is due to the increase in the photoexcited electron-hole pairs between the $\mathrm{p}$ - and $\mathrm{n}$ - layer producing indirect excitons. These excitons have a higher carrier lifetime leading to enhanced performance of the device in photodetection [117]. The inset shows the PL emission for the p-, n-, and pn junction area of the device. An intensity enhancement of $5.9 x$ and $4.5 x$ is observed from the pn-junction at $980 \mathrm{~nm}$ and $900 \mathrm{~nm}$ wavelengths, respectively. This behavior aligns closely with the experimentally tested spectral response of the device. This signifies the generation of new strong absorption peaks in the heterostructurally built material lattice as compared to its natural material form. Such tunability of band absorption can be used for applications in the NIR spectra like LiDAR, gas sensing, photodetectors, optical modulators, and biosensing devices built using the proposed 2DMTS [11], [118]-[120].

\section{Conclusion}

In summary, the family of 2D crystals is continuously growing, both in terms of variety and number of materials as the scientific community progresses rapidly in enhancing the properties in these materials. In this work, we have successfully designed, developed, and demonstrated a novel robust transfer system for 2D materials for on-chip integration for building heterogeneously integrated devices and constructing heterostructures for applications like optoelectronic devices. Using a state-of-the-art micro-stamper and thin bendable film we were able to significantly improve the transfer of 2D materials reliably, with high repeatability, and without incurring any cross-contamination. The latter is a parasitic effect that is often caused by other transfer methods and eliminating this enables chipindustry like repeatability. We also demonstrate a diversity of applications for various active photonic, optoelectronic, and electrical devices, such as a zero-bias photodetector enabling pico-Watt level sensitivity whilst allowing for a dense integration on-chip with a repeatable transfer precision around $500 \mathrm{~nm}$, in the current implementation. This $2 \mathrm{D}$ material transfer system can be upgraded by automation, improving the accuracy, throughput, speed, and reproducibility. This robust and efficient transfer system provides a standard medium for 
expediting research and commercial large-scale integration of 2D materials towards making 2D materials-based integrated devices for a wide range of applications.

\section{SUPPLEMENTARY MATERIAL}

Additional data analysis and simulation results are available in the supplementary document.

\section{CONFLICT OF INTEREST}

The authors have no conflicts to disclose.

\section{ACKNOWLEDGMENT}

VS is supported by AFOSR (FA9550-21-1-0095). A. V. D. acknowledges support through the Material Genome Initiative funding allocated to NIST.

\section{DATA AVAILABLILTY}

The data that supports the findings of this study are available within the article [and its supplementary material].

\section{References}

[1] K. S. Novoselov et al, "Electric field effect in atomically thin carbon films," Science, vol. 306, (5696), pp. 666-669, 2004.

[2] F. Wang et al, "Inter-Flake Quantum Transport of Electrons and Holes in Inkjet-Printed Graphene Devices," Advanced Functional Materials, vol. 31, (5), pp. 2007478, 2021.

[3] M. A. Giambra et al, "Wafer-scale integration of graphene-based photonic devices," ACS Nano, vol. 15, (2), pp. 3171-3187, 2021.

[4] R. Maiti et al, "Loss and coupling tuning via heterogeneous integration of MoS 2 layers in silicon photonics," Optical Materials Express, vol. 9, (2), pp. 751-759, 2019.

[5] K. S. Novoselov et al, "Room-temperature quantum Hall effect in graphene," Science, vol. 315, (5817), pp. 1379, 2007.

[6] C. Patil, R. Maiti and V. J. Sorger, "WS2 monolayer integrated photodetector," in CLEO: QELS_Fundamental Science, 2020, .

[7] R. Maiti et al, "2D material printer: A novel deterministic transfer method for on-chip photonic integration," in 2019 IEEE Research and Applications of Photonics in Defense Conference (RAPID), 2019, .

[8] R. Maiti et al, "Strain-engineered high-responsivity MoTe 2 photodetector for silicon photonic integrated circuits," Nature Photonics, vol. 14, (9), pp. 578-584, 2020. 
[9] H. Agarwal et al, "2D-3D integration of hexagonal boron nitride and a high-K dielectric for ultrafast graphene-based electro-absorption modulators," Nature Communications, vol. 12, (1), pp. 1-6, 2021.

[10] G. Wang et al, "Magneto-optics in transition metal diselenide monolayers," $2 D$ Materials, vol. 2, (3), pp. 034002, 2015.

[11] X. Song, F. Yuan and L. M. Schoop, "The properties and prospects of chemically exfoliated nanosheets for quantum materials in two dimensions," Applied Physics Reviews, vol. 8, (1), pp. 011312, 2021.

[12] A. Castellanos-Gomez, N. Agraït and G. Rubio-Bollinger, "Optical identification of atomically thin dichalcogenide crystals," Appl. Phys. Lett., vol. 96, (21), pp. 213116, 2010.

[13] R. A. Hemnani et al, "2D material printer: a deterministic cross contamination-free transfer method for atomically layered materials," 2D Materials, vol. 6, (1), pp. 015006, 2018.

[14] A. K. Geim and I. V. Grigorieva, "Van der Waals heterostructures," Nature, vol. 499, (7459), pp. 419-425, 2013.

[15] Z. Cai et al, "Chemical vapor deposition growth and applications of two-dimensional materials and their heterostructures," Chem. Rev., vol. 118, (13), pp. 6091-6133, 2018.

[16] Y. S. Ang, H. Y. Yang and L. K. Ang, "Universal scaling laws in Schottky heterostructures based on two-dimensional materials," Phys. Rev. Lett., vol. 121, (5), pp. 056802, 2018.

[17] Y. Gong et al, "Vertical and in-plane heterostructures from WS 2/MoS 2 monolayers," Nature Materials, vol. 13, (12), pp. 1135-1142, 2014.

[18] K. K. Kim, H. S. Lee and Y. H. Lee, "Synthesis of hexagonal boron nitride heterostructures for 2D van der Waals electronics," Chem. Soc. Rev., vol. 47, (16), pp. 6342-6369, 2018.

[19] S. Masubuchi et al, "Autonomous robotic searching and assembly of two-dimensional crystals to build van der Waals superlattices," Nature Communications, vol. 9, (1), pp. 112, 2018.

[20] G. F. Schneider et al, "Wedging transfer of nanostructures," Nano Letters, vol. 10, (5), pp. 1912-1916, 2010.

[21] C. R. Dean et al, "Boron nitride substrates for high-quality graphene electronics," Nature Nanotechnology, vol. 5, (10), pp. 722-726, 2010.

[22] J. Lim et al, "Pattern Pick and Place Method for Twisted Bi-and Multi-Layer Graphene," Materials, vol. 12, (22), pp. 3740, 2019. 
[23] A. Castellanos-Gomez et al, "Deterministic transfer of two-dimensional materials by all-dry viscoelastic stamping," 2D Materials, vol. 1, (1), pp. 011002, 2014.

[24] P. J. Zomer et al, "A transfer technique for high mobility graphene devices on commercially available hexagonal boron nitride," Appl. Phys. Lett., vol. 99, (23), pp. 232104, 2011.

[25] P. Gant et al, "A system for the deterministic transfer of 2D materials under inert environmental conditions," 2D Materials, vol. 7, (2), pp. 025034, 2020.

[26] S. Banerjee, J. Luginsland and P. Zhang, "Interface Engineering of Electrical Contacts," Physical Review Applied, vol. 15, (6), pp. 064048, 2021.

[27] Q. Fu et al, "2D transition metal dichalcogenides: Design, modulation, and challenges in electrocatalysis," Adv Mater, vol. 33, (6), pp. 1907818, 2021.

[28] M. Mannaï and S. Haddad, "Twistronics versus straintronics in twisted bilayers of graphene and transition metal dichalcogenides," Physical Review B, vol. 103, (20), pp. L201112, 2021.

[29] Q. Zhao et al, "An inexpensive system for the deterministic transfer of 2D materials," Journal of Physics: Materials, vol. 3, (1), pp. 016001, 2020.

[30] J. H. Kim et al, "Mechanical properties of two-dimensional materials and their applications," J. Phys. D, vol. 52, (8), pp. 083001, 2018.

[31] R. Zhang and R. Cheung, "Mechanical properties and applications of two-dimensional materials," Two-Dimensional Materials-Synthesis, Characterization and Potential Applications.Rijeka, Crotia: InTech, pp. 219-246, 2016.

[32] P. Cao et al, "Robust and elastic polymer membranes with tunable properties for gas separation," ACS Applied Materials \& Interfaces, vol. 9, (31), pp. 26483-26491, 2017.

[33] R. Addou, L. Colombo and R. M. Wallace, "Surface defects on natural MoS2," ACS Applied Materials \& Interfaces, vol. 7, (22), pp. 11921-11929, 2015.

[34] J. Sun et al, "Impact of impurities on the electrical conduction of anisotropic twodimensional materials," Physical Review Applied, vol. 13, (4), pp. 044063, 2020.

[35] S. Kang et al, "2D semiconducting materials for electronic and optoelectronic applications: potential and challenge," 2D Materials, vol. 7, (2), pp. 022003, 2020.

[36] Y. Mao et al, "Observation of trap-related phenomena in electrical performance of back-gated MoS2 field-effect transistors," Semiconductor Science and Technology, vol. 35, (9), pp. 095023, 2020.

[37] K. Schauble et al, "Uncovering the effects of metal contacts on monolayer MoS2," ACS Nano, vol. 14, (11), pp. 14798-14808, 2020. 
[38] M. Moun, A. Singh and R. Singh, "Study of Electrical Behavior of Metal-

Semiconductor Contacts on Exfoliated MoS2 Flakes," Physica Status Solidi (a), vol. 215, (14), pp. 1800188, 2018.

[39] A. Kumar et al, "Statistical Analysis of Contacts to Synthetic Monolayer MoS2," arXiv Preprint arXiv:2106.08673, 2021.

[40] H. Liu, A. T. Neal and P. D. Ye, "Channel length scaling of MoS2 MOSFETs," ACS Nano, vol. 6, (10), pp. 8563-8569, 2012.

[41] A. T. Neal et al, "Metal contacts to MoS 2: A two-dimensional semiconductor," in 70th Device Research Conference, 2012, .

[42] H. Chang, W. Zhu and D. Akinwande, "On the mobility and contact resistance evaluation for transistors based on MoS2 or two-dimensional semiconducting atomic crystals," Appl. Phys. Lett., vol. 104, (11), pp. 113504, 2014.

[43] N. Kaushik et al, "Schottky barrier heights for Au and Pd contacts to MoS2," Appl. Phys. Lett., vol. 105, (11), pp. 113505, 2014.

[44] de la Rosa, César J Lockhart et al, "Insight on the characterization of MoS2 based devices and requirements for logic device integration," ECS Journal of Solid State Science and Technology, vol. 5, (11), pp. Q3072, 2016.

[45] H. Liu et al, "Fundamentals in MoS2 transistors: dielectric, scaling and metal contacts," ECS Transactions, vol. 58, (7), pp. 203, 2013.

[46] A. Pacheco-Sanchez and D. Jiménez, "A contact resistance extraction method of 2DFET technologies without test structures," in 2021 13th Spanish Conference on Electron Devices (CDE), 2021, .

[47] I. P. Handayani et al, "Optical and electrical characterization of WS2 multilayer on flexible PET substrate," Materials Research Express, vol. 8, (2), pp. 026405, 2021.

[48] M. Long et al, "Progress, challenges, and opportunities for 2D material based photodetectors," Advanced Functional Materials, vol. 29, (19), pp. 1803807, 2019.

[49] T. Dong, J. Simões and Z. Yang, "Flexible photodetector based on 2D materials:

processing, architectures, and applications," Advanced Materials Interfaces, vol. 7, (4), pp. 1901657, 2020.

[50] M. Gibertini et al, "Magnetic 2D materials and heterostructures," Nature Nanotechnology, vol. 14, (5), pp. 408-419, 2019.

[51] K. Zhou, J. Jiang and L. Ding, "2D transition metal dichalcogenides for neuromorphic vision system," Journal of Semiconductors, vol. 42, (9), pp. 090203-3, 2021. 
[52] G. Cao et al, "2D material based synaptic devices for neuromorphic computing," Advanced Functional Materials, vol. 31, (4), pp. 2005443, 2021.

[53] C. Liu et al, "Silicon/2D-material photodetectors: from near-infrared to mid-infrared," Light: Science \& Applications, vol. 10, (1), pp. 1-21, 2021.

[54] $\mathrm{H}$. Chen et al, "Logic gates based on neuristors made from two-dimensional materials," Nature Electronics, pp. 1-6, 2021.

[55] L. Mennel et al, "Ultrafast machine vision with 2D material neural network image sensors," Nature, vol. 579, (7797), pp. 62-66, 2020.

[56] A. Reserbat-Plantey et al, "Quantum nanophotonics in two-dimensional materials," ACS Photonics, vol. 8, (1), pp. 85-101, 2021.

[57] S. Bae et al, "Integration of bulk materials with two-dimensional materials for physical coupling and applications," Nature Materials, vol. 18, (6), pp. 550-560, 2019.

[58] P. Sutter, S. Wimer and E. Sutter, "Chiral twisted van der Waals nanowires," Nature, vol. 570, (7761), pp. 354-357, 2019.

[59] D. Kim et al, "Two-dimensional phase-engineered 1T'-and 2H-MoTe2-based nearinfrared photodetectors with ultra-fast response," J. Alloys Compounds, vol. 789, pp. 960965, 2019.

[60] Y. Bie et al, "A MoTe 2-based light-emitting diode and photodetector for silicon photonic integrated circuits," Nature Nanotechnology, vol. 12, (12), pp. 1124-1129, 2017.

[61] P. Ma et al, "Fast MoTe2 waveguide photodetector with high sensitivity at telecommunication wavelengths," ACS Photonics, vol. 5, (5), pp. 1846-1852, 2018.

[62] $\mathrm{H}$. Huang et al, "Ferroelectric polymer tuned two dimensional layered MoTe2 photodetector," RSC Advances, vol. 6, (90), pp. 87416-87421, 2016.

[63] T. J. Octon et al, "Fast High-Responsivity Few-Layer MoTe2 Photodetectors," Advanced Optical Materials, vol. 4, (11), pp. 1750-1754, 2016.

[64] $\mathrm{H}$. Huang et al, "Highly sensitive visible to infrared MoTe2 photodetectors enhanced by the photogating effect," Nanotechnology, vol. 27, (44), pp. 445201, 2016.

[65] C. S. Patil, "2D Material Integration with Integrated Photonics: Waveguides, MicroRing Resonators and Photodetectors." M.S., The George Washington University, United States -- District of Columbia, 2019.

[66] L. N. Tripathi et al, "Spontaneous emission enhancement in strain-induced WSe2 monolayer-based quantum light sources on metallic surfaces," ACS Photonics, vol. 5, (5), pp. 1919-1926, 2018. 
[67] S. B. Desai et al, "Strain-induced indirect to direct bandgap transition in multilayer WSe2," Nano Letters, vol. 14, (8), pp. 4592-4597, 2014.

[68] Y. Luo et al, "Exciton dipole orientation of strain-induced quantum emitters in WSe2," Nano Letters, vol. 20, (7), pp. 5119-5126, 2020.

[69] B. Amin, T. P. Kaloni and U. Schwingenschlögl, "Strain engineering of WS 2, WSe 2, and WTe 2," Rsc Advances, vol. 4, (65), pp. 34561-34565, 2014.

[70] X. Zhang et al, "Phonon and Raman scattering of two-dimensional transition metal dichalcogenides from monolayer, multilayer to bulk material," Chem. Soc. Rev., vol. 44, (9), pp. 2757-2785, 2015.

[71] S. Chen et al, "Helicity-resolved Raman scattering of MoS2, MoSe2, WS2, and WSe2 atomic layers," Nano Letters, vol. 15, (4), pp. 2526-2532, 2015.

[72] D. Zambrano et al, "Spin and Valley Filter Based on Two-Dimensional W Se 2 Heterostructures," Physical Review Applied, vol. 15, (3), pp. 034069, 2021.

[73] K. Lin et al, "Twist-angle engineering of excitonic quantum interference and optical nonlinearities in stacked 2D semiconductors," Nature Communications, vol. 12, (1), pp. 17, 2021.

[74] J. Henriques, M. M. Quintela and N. Peres, "Theoretical model of the polarizability due to transitions between exciton states in transition metal dichalcogenides: application to WSe 2," Josa B, vol. 38, (7), pp. 2065-2074, 2021.

[75] Y. Cho, J. Huang and C. W. Wong, "Collective excitations in 2D atomic layers: Recent perspectives," Appl. Phys. Lett., vol. 116, (2), pp. 020501, 2020.

[76] Y. Liu et al, "Van der Waals heterostructures and devices," Nature Reviews Materials, vol. 1, (9), pp. 1-17, 2016.

[77] W. J. Yu et al, "Vertically stacked multi-heterostructures of layered materials for logic transistors and complementary inverters," Nature Materials, vol. 12, (3), pp. 246-252, 2013.

[78] S. J. Haigh et al, "Cross-sectional imaging of individual layers and buried interfaces of graphene-based heterostructures and superlattices," Nature Materials, vol. 11, (9), pp. 764-767, 2012.

[79] L. Britnell et al, "Field-effect tunneling transistor based on vertical graphene heterostructures," Science, vol. 335, (6071), pp. 947-950, 2012.

[80] Y. Saito, T. Nojima and Y. Iwasa, "Highly crystalline 2D superconductors," Nature Reviews Materials, vol. 2, (1), pp. 1-18, 2016. 
[81] C. Choi et al, "Enhanced interlayer neutral excitons and trions in trilayer van der Waals heterostructures," Npj 2D Materials and Applications, vol. 2, (1), pp. 1-9, 2018.

[82] Y. Cao et al, "Unconventional superconductivity in magic-angle graphene superlattices," Nature, vol. 556, (7699), pp. 43-50, 2018.

[83] D. R. Klein et al, "Probing magnetism in 2D van der Waals crystalline insulators via electron tunneling," Science, vol. 360, (6394), pp. 1218-1222, 2018.

[84] P. Rivera et al, "Valley-polarized exciton dynamics in a 2D semiconductor heterostructure," Science, vol. 351, (6274), pp. 688-691, 2016.

[85] F. Withers et al, "Light-emitting diodes by band-structure engineering in van der Waals heterostructures," Nature Materials, vol. 14, (3), pp. 301-306, 2015.

[86] C. Lee et al, "Atomically thin p-n junctions with van der Waals heterointerfaces," Nature Nanotechnology, vol. 9, (9), pp. 676-681, 2014.

[87] H. Fang et al, "Strong interlayer coupling in van der Waals heterostructures built from single-layer chalcogenides," Proceedings of the National Academy of Sciences, vol. 111, (17), pp. 6198-6202, 2014.

[88] W. J. Yu et al, "Highly efficient gate-tunable photocurrent generation in vertical heterostructures of layered materials," Nature Nanotechnology, vol. 8, (12), pp. 952-958, 2013.

[89] R. Wang et al, "2d or not 2d: strain tuning in weakly coupled heterostructures," Advanced Functional Materials, vol. 28, (14), pp. 1705901, 2018.

[90] S. Biswas et al, "Nonlinear optical properties and temperature dependent photoluminescence in hBN-GO heterostructure 2D material," The Journal of Physical Chemistry C, vol. 121, (14), pp. 8060-8069, 2017.

[91] G. lannaccone et al, "Quantum engineering of transistors based on 2D materials heterostructures," Nature Nanotechnology, vol. 13, (3), pp. 183-191, 2018.

[92] J. Yu et al, "Designing the Bending Stiffness of 2D Material Heterostructures," Adv Mater, vol. 33, (9), pp. 2007269, 2021.

[93] K. S. Novoselov et al, "2D materials and van der Waals heterostructures," Science, vol. 353, (6298), 2016.

[94] F. Wang et al, "Tunable GaTe-MoS2 van der Waals $p-n$ junctions with novel optoelectronic performance," Nano Letters, vol. 15, (11), pp. 7558-7566, 2015.

[95] X. Yuan et al, "Wafer-scale arrayed pn junctions based on few-layer epitaxial GaTe," Nano Research, vol. 8, (10), pp. 3332-3341, 2015. 
[96] K. Kang et al, "Layer-by-layer assembly of two-dimensional materials into wafer-scale heterostructures," Nature, vol. 550, (7675), pp. 229-233, 2017.

[97] B. Yao et al, "Gate-tunable frequency combs in graphene-nitride microresonators," Nature, vol. 558, (7710), pp. 410-414, 2018.

[98] L. Lv et al, "Design and tailoring of two-dimensional Schottky, PN and tunnelling junctions for electronics and optoelectronics," Nanoscale, vol. 13, (14), pp. 6713-6751, 2021.

[99] M. Mathew et al, "A review on mechanisms and recent developments in pn heterojunctions of 2D materials for gas sensing applications," J. Mater. Sci., pp. 1-30, 2021.

[100] Y. Chen et al, "Two-dimensional WSe2/SnSe pn junctions secure ultrahigh thermoelectric performance in n-type $\mathrm{Pb} / \mathrm{l}$ Co-doped polycrystalline SnSe," Materials Today Physics, vol. 16, pp. 100306, 2021.

[101] B. Gharekhanlou and S. Khorasani, "Current-voltage characteristics of graphane pn junctions," IEEE Trans. Electron Devices, vol. 57, (1), pp. 209-214, 2009.

[102] $\mathrm{H}$. Ilatikhameneh et al, "Dramatic impact of dimensionality on the electrostatics of PN junctions and its sensing and switching applications," IEEE Transactions on Nanotechnology, vol. 17, (2), pp. 293-298, 2018.

[103] M. Buscema et al, "Photovoltaic effect in few-layer black phosphorus PN junctions defined by local electrostatic gating," Nature Communications, vol. 5, (1), pp. 1-6, 2014.

[104] A. Nipane et al, "Electrostatics of lateral pn junctions in atomically thin materials," J. Appl. Phys., vol. 122, (19), pp. 194501, 2017.

[105] M. Vemula, S. Veeralingam and S. Badhulika, "Hybrid 2D/0D SnSe2-SnO2 vertical junction based high performance broadband photodetector," J. Alloys Compounds, pp. 160826, 2021.

[106] D. Wu et al, "Ultrabroadband and High-Detectivity Photodetector Based on WS2/Ge Heterojunction through Defect Engineering and Interface Passivation," ACS Nano, 2021.

[107] B. Yao et al, "Broadband gate-tunable terahertz plasmons in graphene heterostructures," Nature Photonics, vol. 12, (1), pp. 22-28, 2018.

[108] Z. Chen, J. Biscaras and A. Shukla, "A high performance graphene/few-layer InSe photo-detector," Nanoscale, vol. 7, (14), pp. 5981-5986, 2015.

[109] S. R. Tamalampudi et al, "High performance and bendable few-layered InSe photodetectors with broad spectral response," Nano Letters, vol. 14, (5), pp. 2800-2806, 2014. 
[110] W. Zhang et al, "Role of metal contacts in high-performance phototransistors based on WSe2 monolayers," ACS Nano, vol. 8, (8), pp. 8653-8661, 2014.

[111] D. A. Nguyen et al, "Highly enhanced photoresponsivity of a monolayer WSe2 photodetector with nitrogen-doped graphene quantum dots," ACS Applied Materials \& Interfaces, vol. 10, (12), pp. 10322-10329, 2018.

[112] O. Lopez-Sanchez et al, "Ultrasensitive photodetectors based on monolayer MoS 2," Nature Nanotechnology, vol. 8, (7), pp. 497-501, 2013.

[113] W. Feng et al, "Ultrahigh photo-responsivity and detectivity in multilayer InSe nanosheets phototransistors with broadband response," Journal of Materials Chemistry $C$, vol. 3, (27), pp. 7022-7028, 2015.

[114] M. Li et al, "High mobilities in layered InSe transistors with indium-encapsulationinduced surface charge doping," Adv Mater, vol. 30, (44), pp. 1803690, 2018.

[115] R. Cheng et al, "Electroluminescence and photocurrent generation from atomically sharp WSe2/MoS2 heterojunction p-n diodes," Nano Letters, vol. 14, (10), pp. 5590-5597, 2014.

[116] C. Patil et al, "Self-Driven Highly Responsive PN Junction InSe Heterostructure Near-Infrared Light Detector," arXiv Preprint arXiv:2108.11803, 2021.

[117] Y. Wang et al, "Molecular Doping of 2D Indium Selenide for Ultrahigh Performance and Low-Power Consumption Broadband Photodetectors," Advanced Functional Materials, pp. 2103353, 2021.

[118] D. Kaiser et al, "pH sensors based on amino-terminated carbon nanomembrane and single-layer graphene van der Waals heterostructures," Applied Physics Reviews, vol. 8, (3), pp. 031410, 2021.

[119] C. Chiang et al, "Memory applications from 2D materials," Applied Physics Reviews, vol. 8, (2), pp. 021306, 2021.

[120] A. Asghari et al, "Fast, accurate, point-of-care COVID-19 pandemic diagnosis enabled through advanced lab-on-chip optical biosensors: Opportunities and challenges," Applied Physics Reviews, vol. 8, (3), pp. 031313, 2021. 